\title{
An atom counting and electrophilicity based QSTR approach
}

\author{
P K CHATTARAJ, ${ }^{1, *}$ D R ROY,${ }^{1}$ S GIRI, ${ }^{1}$ S MUKHERJEE, ${ }^{1}$ V SUBRAMANIAN,${ }^{2, *}$ \\ R PARTHASARATHI, ${ }^{2}$ P BULTINCK ${ }^{3, *}$ and S VAN DAMME ${ }^{3}$ \\ ${ }^{1}$ Department of Chemistry, Indian Institute of Technology, Kharagpur 721302 \\ ${ }^{2}$ Chemical Laboratory, Central Leather Research Institute, Adyar, Chennai 600020 \\ ${ }^{3}$ Department of Inorganic and Physical Chemistry, Ghent University, Krijgslaan 281, B-9000 Gent, Belgium \\ e-mail:pkc@chem.iitkgp.ernet.in; subuchem@hotmail.com; Patrick.Bultinck@ugent.be
}

MS received 28 April 2007; accepted 17 July 2007

\begin{abstract}
Quantitative-structure-toxicity-relationship (QSTR) models are developed for predicting the toxicity $\left(\mathrm{pIGC}_{50}\right)$ of 252 aliphatic compounds on Tetrahymena pyriformis. The single parameter models with a simple molecular descriptor, the number of atoms in the molecule, provide reasonable results. Better QSTR models with two parameters result when global electrophilicity is used as the second descriptor. In order to tackle both charge- and frontier-controlled reactions the importance of the local electro (nucleo) philicities and atomic charges is also analysed.
\end{abstract}

Keywords. Atom counting; QSTR; electrophilicity; conceptual DFT; Tetrahymena pyriformis.

\section{Introduction}

Ever since the power of Quantitative-structure-activity-relationship (QSAR) based techniques has been highlighted, several descriptors have been proposed from time to time in developing QSAR models ${ }^{1-8}$ for understanding various aspects of pharmacological sciences including drug design and the possible ecotoxicological characteristics of the drug molecules. Specific quantitative-structure toxicity-relationship (QSTR) models have also been developed. In these studies the toxicity of various chemicals have been understood via corresponding molecular structures. An extensive research has been carried out ${ }^{9-16}$ in understanding the toxicological effects of several aliphatic compounds on ciliated protozoa called Tetrahymena pyriformis. Both European Union and US Environmental Protection Agency require reliable toxicity data set for various classes of living systems like primary producers, invertebrates and vertebrates. This information is used for QSAR/QSTR as well as regulatory purposes. The ciliated protozoa, Tetrahymena pyriformis has been considered to be ideal for the associated laboratory research. In this ciliate species, diverse endpoints can be used to originate the cytotoxic effects and xenobiotics. Experimental deter-

*For correspondence mination of toxicological and biochemical endpoints is a difficult task. Hence, QSAR/QSTR modelling of the toxicity of aliphatic compounds on the T. pyriformis is of vital importance in investigation of its toxicity in terms of its inhibitory growth concentration (IGC). A multitude of QSTR models exist which analyse the associated toxicity behaviour. Quantum chemical descriptors ${ }^{17-20}$ have also been used for this purpose and they have been proved to be versatile and reliable.

Toxicity analyses of a diverse class of systems have been carried out using conceptual density functional theory (DFT) based reactivity/selectivity descriptors. Possibility of electron transfer between a toxic molecule and a biosystem has been considered to be one of the major reasons of toxic behaviour of these molecules. Accordingly the related descriptors like electron affinity, ionization potential, planarity, electrophilicity, etc. have been turned out to be useful QSTR descriptors. Experimental toxicity values of a wide variety of polyaromatic hydrocarbons like polychlorinated dibenzofurans (PCDFs), polychlorinated biphenyls (PCBs), polychlorinated dibenzo-pdioxins (PCDDs) and chlorophenols (CP), several aliphatic and aromatic toxic molecules have been shown to correlate very well ${ }^{20-30}$ with the corresponding toxicity values estimated using various conceptual DFT descriptors especially global and local electrophilicities. $^{31-33}$ 
Several researchers ${ }^{9-16}$ have studied the toxicological behaviour of various compounds on T. pyriformis. They have highlighted the importance of the studies as well as the possibility of constructing a large number of QSTR models with a varied range of success and the difficulty in computation. A stateof-the-art QSTR model has been developed by Schultz et $a l^{13}$. Toxicity of a large number of aliphatic compounds on $T$. pyriformis has been studied $^{13}$ through QSTR models developed ${ }^{13}$ in terms of $\log \mathrm{P}$ and the lowest unoccupied molecular orbital energy $\left(E_{\text {LUMO }}\right)$ whereas the effect of several aromatic compounds on the same system has been analysed $^{14}$ in terms of $\log P, E_{\mathrm{LUMO}}$ and maximum acceptor superdelocalizability $\left(A_{\max }\right)$. In both cases the models are found to be robust. We have shown ${ }^{20,25}$ that global and local electrophilicities are useful descriptors of toxicity prediction. In the present work we propose to develop QSTR models for toxicity of several aliphatic compounds on T. pyriformis, using the number of atoms present in the molecule, which can be obtained very easily. Section 2 provides the theoretical background whereas the computational details are provided in $\S 3$. Results and discussion are provided in $\S 4$ and finally, $\S 5$ gives some concluding remarks.

\section{Theoretical background}

We consider the number of atoms in a molecule to be a valid descriptor of its toxic nature. For a given group of molecules the number of electrons $(N)$ is expected to scale as the number of atoms present $\left(N_{a}\right)$. Molecules with larger $N_{a}$ values are supposed to have larger molecular weights implying larger $\log P$ values. That in turn will provide larger toxicity values. For simplicity, we consider the number of carbon atoms $\left(N_{C}\right)$ as the variable and for the set of molecules with a constant $N_{C}$ we may choose the number of non-hydrogenic atoms $\left(N_{\mathrm{NH}}\right)$ as the descriptor. Related descriptors have been used in the past. ${ }^{34 a}$ Its usefulness has also been demonstrated in developing QSAR model for the biological activities of sex hormones ${ }^{34 \mathrm{~b}}$ and new QSPR models for boiling point of alcohols, enthalpy of vaporization and $\log \mathrm{P}$ of PCBs and chloroanisoles and $p K a$ values for various acids and alcohols. ${ }^{34 \mathrm{c}}$

In order to have a complete analysis we also check the nature of the model where electrophilicity $(\omega)$ is used as an additional descriptor, which has been shown ${ }^{20,25}$ to be a reliable descriptor of biological activity ${ }^{19}$ and toxicity. ${ }^{20,25}$ The electrophilicity is defined $\mathrm{as}^{31,32}$

$$
\omega=\frac{\mu^{2}}{2 \eta},
$$

where $\mu=-(I+A) / 2$ and $\eta=(I-A) / 2$ are the electronic chemical potential and hardness respectively. $I$ and $A$ being the ionization potential and electron affinity respectively.

It has also been shown ${ }^{20,25}$ that apart from global electrophilic power the local electro (nucleo) philicity is important in understanding the possible charge transfer between a toxin and a receptor. The philicity at an atom $k$ of the molecule is defined as ${ }^{33}$

$$
\omega_{k}^{\alpha}=\omega \cdot f_{k}^{\alpha},
$$

where $\left\{f_{k}^{\alpha}\right\}$ are the condensed-to-atom- $k$ Fukui functions calculated in terms of the electronic population $q_{k}$ and $\alpha=+,-$ and 0 refers to nucleophilic, electrophilic and radical attacks respectively. The condensed Fukui functions are given by ${ }^{35}$

$$
\begin{aligned}
& f_{k}^{+}=q_{k}(N+1)-q_{k}(N), \\
& f_{k}^{-}=q_{k}(N)-q_{k}(N-1), \\
& f_{k}^{0}=\left[q_{k}(N+1)-q_{k}(N-1)\right] / 2,
\end{aligned}
$$

Since the Fukui function based descriptors are ideally suited for soft-soft-frontier-controlled reactions and the atomic charges $\left(Q_{k}\right)$ in a molecule are known to be appropriate local descriptors in analysing essentially charged-controlled reactions between a hard nucleophile and a hard electrophile ${ }^{36-38}$ we also consider the latter in our analysis.

Comparing the electronegativity values of 13 sets of aliphatic compounds whose toxic nature towards T. pyriformis is known, ${ }^{9-16}$ with those of various nucleic acid bases (adenine, thymine, guanine, cytosine and urasil) and DNA base pairs (GCWC and ATH) it was observed ${ }^{20}$ that there are nine groups of electron acceptors (saturated alcohols, diols, halogenated alcohols, mono- and di- esters, carboxylic and halogenated acids, aldehydes and ketones) and four groups of electron donors (unsaturated alcohols, $\alpha$-acetylinic alcohols, amino alcohols and amines). For the former group $\omega_{\max }^{+}$and for the latter group $\omega_{\max }^{-}$are considered to be $\mathrm{b}^{20}$ appropriate descriptors where $\omega_{\max }^{\alpha}$ refers to the $\omega_{k}^{\alpha}$ value at the site where it is maximum. For the hard interactions, $Q_{k}^{\max }$ is considered to be the 
proper descriptor where $k$ is the site with the maximum value of the magnitude of the charge (positive for the acceptors and negative for the donors).

\section{Computational details}

Geometries of all the 252 aliphatic molecules (acceptors-171, donors-81) corresponding to the 13 groups are optimized at the Hatree-Fock level with 6$311 \mathrm{G}^{*}$ basis set using the Gaussian $03^{39}$ program. These molecules were tested before ${ }^{20,25}$ for correlating their experimental $\log \left(\mathrm{IGC}_{50}{ }^{-1}\right)$ values ${ }^{10}$ against $T$. pyriformis with the corresponding values calculated in terms of global and local electrophilicities.

Equations (1)-(5) are used to calculate the global and local electrophilicities. Necessary population and charges are calculated using the natural population analysis (NPA) scheme. Single point calculations are done for the $(N \pm 1)$ - electron systems with the $N$ electron molecule geometry.

Initially we have performed an exhaustive statistical analysis in which the systematic search is carried out to find out the most potent descriptors from the statistically significant relationships between the toxicity and a selection of one, two or three descriptors out of the six available descriptors $\left(N_{\mathrm{C}}, \mathrm{N}_{\mathrm{NH}}, \omega\right.$, $\omega_{\max }^{+}, \omega_{\max }^{-}$and $\left.Q_{k}^{\max }\right)$. The analysis is performed using in-house software. In order to minimize the effect of multi-collinearity and to avoid redundancy, the descriptor set is first pre-evaluated with unsupervised forward selection. This selection is a variable elimination technique where variables are physically removed from the data set. Variables are eliminated for two reasons. First, they are eliminated if they have a small variance, below some threshold value. The second reason for variable removal is the existence of redundancy (exact linear dependencies between subsets of the variables) and multicollinearity (high multiple correlations between subsets of the variables) in QSAR data sets. Multicollinearity and redundancy may result in highly unstable estimates for regression coefficients, because their values may change enormously when variables are added or deleted to the regression. Both these features are assessed by inspecting the multiple correlations within the relevant subsets of descriptors. For a detailed overview of the UFS algorithm we refer to references 40 and 41. As regression technique the multiple linear regression is preferred over principal component regression or partial least squares regression, because of the ease of interpretation of the out- come. The following statistical criteria of the models are noted: $R, R$-square $\left(R^{2}\right)$, adjusted R-square $\left(R_{\mathrm{adj}}^{2}\right)$ and the standard errors of the estimate are measured to confirm a good fit of the data to the regression line. Internal validation is conducted with leave-one-out cross-validation and is given by $Q^{2}$. The significance of this value is estimated by Y-randomisation.

$R_{\text {adj }}^{2}$ is defined as:

$$
R_{\mathrm{adj}}^{2}=1-\left(1-R^{2}\right)\{(N-1) /(N-p-1)\},
$$

where $N$ is the sample size and $p$ is the number of terms in the model not counting the constant (i.e. the number of independents).

The cross-validated standard coefficient, $Q^{2}$, is defined as follows:

$$
Q^{2}=1-\frac{\sum_{Y}\left(Y_{\text {predicted }}-Y_{\text {observed }}\right)^{2}}{\sum_{Y}\left(Y_{\text {observed }}-Y_{\text {mean }}\right)^{2}},
$$

where $Y_{\text {predicted }}, Y_{\text {observed }}$, and $Y_{\text {mean }}$ are the predicted, observed, and mean values of the target property respectively. $\sum\left(Y_{\text {predicted }}-Y_{\text {observed }}\right)^{2}$ is the predictive error sum of squares (PRESS).

$F$-test (Fisher value: level of statistical significance) is defined as:

$$
F=R^{2}(N-p-1) /\left\{p\left(1-R^{2}\right)\right\},
$$

where $N$ is the sample size and $p$ is the number of terms in the model not counting the constant (i.e. the number of independents).

A mechanistic interpretation can be deduced from the output, by using the coefficients (b). These are descriptors calculated from scaled data values. This gives the opportunity to evaluate the descriptors in relation to each other. Outliers are detected graphically in the regression diagnostic plot.

Each statistical analysis is preceded by an analysis of the dataset. A graphical analysis of the residuals (residual plot, normal probability plot and regression plot) permits the user to confirm if the dataset is suitable for a multiple linear regression.

\section{Results and discussion}

A systematic search is performed to investigate all the possible combinations of one-, two- and threeparameter models out of six chosen possibilities, viz. $N_{\mathrm{C}}, N_{\mathrm{NH}}, \omega, \omega_{\max }^{+}, \omega_{\max }^{-}$and $Q_{k}^{\max }$ to obtain the most useful and statistically significant descriptors in pre- 
dicting the toxicity of the various aliphatic compounds considered in the study. Various plots and the model summary with the best possible combination of three descriptors are shown (see Supporting Information (SI): Figures S1-S13) to see the relative importance of the different descriptors. They are presented sequentially. Now we will investigate for each set of molecules whether we can obtain threeparameter models which behave better/similar than/to the already obtained one-and two-parameter models. The best three-parameter model is chosen based on the highest $R^{2}$ value. To compare three-parameter models with one-and two-parameter models, we cannot use the $R^{2}$ value. The value of $R^{2}$ can generally be increased by adding additional descriptor variables to the model, even if the added variable does not contribute to reduce the unexplained variance of the dependent variable. This can be avoided by using another statistical parameter - the so-called adjusted $R^{2}\left(R_{\text {adj }}^{2}\right)$.

Before any regression analysis can take place, we have to check the data set on a few principal assumptions. These assumptions justify the use of linear regression models for purposes of prediction:

Independence: The response variables are not dependent on one another.

Normality: The response variables have to be distributed normally. This check happens in a normal probability plot, and if the distribution is normal, the points on this plot should fall close enough to the diagonal line.

Linearity: The dependent variables are linearly related to the independent variables, i.e. the relationship is a straight line.

If any of these assumptions is violated, then the insights yielded by a regression model may be ineffective or seriously biased or misleading. The characteristics of the data sets are checked visually. For a detailed overview of these assumptions and their visualization, the reader is referred to the reference 42. For the purpose of this article it is sufficient to look at the graphics (a), (c) and (d) for each of the data sets (see SI: Figures S1-S13). The graphic (a) has to be a scattered plot of points around zero and the graphics (c) and (d) have to be a straight line of points through the origin (see SI: Figures S1-S13). It has been noticed that for most of the thirteen data sets considered in our study, these characteristics are fulfilled.

The performance of the multiple linear regression is summarized in a few statistical parameters. The most important ones for this purpose are $R_{\mathrm{adj}}^{2}$, the standard error of the estimate, $Q^{2}$ and the F-ratio. Each of these terms is explained in the previous section and their behaviour can be found in statistical literature. If we encounter a model which does not behave well for one of these parameters, the model has to be rejected. Based on these four statistical parameters, each of the models can be accepted as statistically significant models.

The fact that each of these 3-parameter models is statistically significant does not mean that they behave better then the corresponding 1- or 2-parameter models. In view of the behaviour of these models one has to look at three parameters. First of all, as mentioned before, one has to compare the adjusted $R^{2}$ of the three-parameter model with those of the one- or two-parameter models. All of the threeparameter models concerning $R^{2}\left(R^{2}, R_{\mathrm{adj}}^{2}, R_{\mathrm{cv}}^{2}\right)$ behave slightly better than the corresponding one- or two-parameter models, except the model for the set of $\alpha$-acetylenic alcohols, in which the $R_{\mathrm{adj}}^{2}$ and $R_{\mathrm{cv}}^{2}$ behave worse than the two-parameter model (see SI: table S1). This may be a case of overfitting. ${ }^{43}$ For the saturated alcohols and diesters, $R_{\mathrm{adj}}^{2}$ and $R_{\mathrm{cv}}^{2}$ of the three-parameter models increase slightly by adding one descriptor to their two-parameter models (see SI: table S1). Since it is better to have a model with as least as possible descriptors, according the Principle of Parsimony, ${ }^{44}$ we prefer for these sets the two-parameter models.

Three parameter models are only better than the two-parameter models, if the three parameters used are statistically significant. The in-house built statistical software conducts this test of significance with a student's $t$-test. In the case of halogenated acids, aldehydes and amino alcohols, their three-parameter models contain one/two non-significant descriptors (See SI: table S1). The remaining models can be used for predictive purposes, only if they do not fail on the Y-randomisation test. As can be seen on the graphs (e), this concerns only the three-parameter models of the carboxylic acids, monoesters, unsaturated alcohols and aliphatic amines (see SI: table S1 and figures S1-S13). The fact that the remaining models of the halogenated alcohols, the diols and the ketones do not provide good Y-randomisation test results might be originating from the small number of molecules (respectively 11, 10 and 15) for the number of descriptors used. ${ }^{45}$

It is important to note that $\omega_{\max }^{+}$and $\omega_{\max }^{-}$respectively appear in the electron acceptor and donor sets, 
Table 1. Electrophilicity $(\omega)$, maximum atomic charge $\left(Q_{k}^{\max }\right)$, number of carbon atoms $(N c), \log P$ along with the experimental and calculated values of $\log \left(\mathrm{IGC}_{50}{ }^{-1}\right)$ for the complete set of aliphatic acceptor compounds with Tetrahymena pyriformis.

\begin{tabular}{|c|c|c|c|c|c|c|c|}
\hline \multirow[b]{2}{*}{ Molecules } & \multirow[b]{2}{*}{$\omega$} & \multirow[b]{2}{*}{$Q_{k}^{\max }$} & \multirow[b]{2}{*}{$N c$} & \multirow[b]{2}{*}{$\log P^{*}$} & \multicolumn{3}{|c|}{$\mathrm{pIGC}_{50}$} \\
\hline & & & & & Experiment* & Calc. $(N C)$ & Calc. $(N c, \omega)$ \\
\hline \multicolumn{8}{|l|}{ Diols } \\
\hline$(+/-)-1,2-$ Butanediol & $0 \cdot 8999$ & $0 \cdot 4652$ & 4 & $-0 \cdot 53$ & $-2 \cdot 0482$ & $-2 \cdot 2868$ & $-2 \cdot 1479$ \\
\hline$(+/-)-1,3$-Butanediol & 0.9286 & $0 \cdot 4488$ & 4 & $-1 \cdot 38$ & $-2 \cdot 3013$ & $-2 \cdot 2868$ & $-2 \cdot 5044$ \\
\hline 1,4-Butanediol & $0 \cdot 8915$ & $0 \cdot 4492$ & 4 & $-0 \cdot 83$ & $-2 \cdot 2365$ & $-2 \cdot 2868$ & $-2 \cdot 0435$ \\
\hline 1,2-Pentanediol & $0 \cdot 8907$ & $0 \cdot 4652$ & 5 & $0 \cdot 00$ & -1.6269 & $-1 \cdot 8371$ & $-1 \cdot 6782$ \\
\hline 1,5-Pentanediol & $0 \cdot 9132$ & 0.4487 & 5 & $-0 \cdot 64$ & $-1 \cdot 9344$ & $-1 \cdot 8371$ & $-1 \cdot 9577$ \\
\hline 2-Methyl-2,4-pentanediol & $0 \cdot 9200$ & $0 \cdot 4463$ & 6 & $-0 \cdot 68$ & $-1 \cdot 9531$ & $-1 \cdot 3874$ & $-1 \cdot 6868$ \\
\hline$(+/-)-1,2-$ Hexanediol & $0 \cdot 8887$ & $0 \cdot 4652$ & 6 & $0 \cdot 53$ & $-1 \cdot 2669$ & $-1 \cdot 3874$ & $-1 \cdot 2979$ \\
\hline 1,6-Hexanediol & $0 \cdot 9027$ & $0 \cdot 4487$ & 6 & $-0 \cdot 11$ & -1.4946 & $-1 \cdot 3874$ & -1.4719 \\
\hline 1,2-Decanediol & $0 \cdot 8640$ & $0 \cdot 4651$ & 10 & $2 \cdot 64$ & $0 \cdot 7640$ & $0 \cdot 4113$ & $0 \cdot 4305$ \\
\hline 1,10-Decanediol & $0 \cdot 8597$ & $0 \cdot 4484$ & 10 & $2 \cdot 01$ & $0 \cdot 2240$ & $0 \cdot 4113$ & $0 \cdot 4839$ \\
\hline \multicolumn{8}{|l|}{ Halogenated alcohol } \\
\hline 2-Bromoethanol & 0.9418 & 0.4575 & 2 & $0 \cdot 18$ & $-0 \cdot 8457$ & $-1 \cdot 3706$ & -0.9446 \\
\hline 2-Chloroethanol & $1 \cdot 0417$ & $0 \cdot 4578$ & 2 & $-0 \cdot 06$ & $-1 \cdot 4174$ & $-1 \cdot 3706$ & $-1 \cdot 5727$ \\
\hline 1-Chloro-2-propanol & $1 \cdot 0170$ & 0.4549 & 3 & $0 \cdot 14$ & $-1 \cdot 492$ & $-1 \cdot 0434$ & $-1 \cdot 2191$ \\
\hline 3-Chloro-1-propanol & $1 \cdot 0101$ & $0 \cdot 4525$ & 3 & $0 \cdot 50$ & $-1 \cdot 3992$ & $-1 \cdot 0434$ & $-1 \cdot 1758$ \\
\hline 4-Chloro-1-butanol & $0 \cdot 9570$ & $0 \cdot 4514$ & 4 & $0 \cdot 85$ & -0.7594 & -0.7163 & $-0 \cdot 6437$ \\
\hline 3-Chloro-2,2-dimethyl-1-propanol & $0 \cdot 9843$ & $0 \cdot 4553$ & 5 & $0 \cdot 81$ & -0.7822 & $-0 \cdot 3892$ & $-0 \cdot 6171$ \\
\hline 6-Chloro-1-hexanol & $0 \cdot 9417$ & $0 \cdot 4497$ & 6 & $1 \cdot 59$ & $-0 \cdot 2726$ & $-0 \cdot 0621$ & $-0 \cdot 151$ \\
\hline 8-Chloro-1-octanol & $0 \cdot 9278$ & $0 \cdot 4490$ & 8 & $2 \cdot 65$ & 0.4878 & 0.5921 & $0 \cdot 3329$ \\
\hline 6-Bromo-1-hexanol & $0 \cdot 8636$ & $0 \cdot 4497$ & 6 & $1 \cdot 73$ & $0 \cdot 0074$ & $-0 \cdot 0621$ & $0 \cdot 3399$ \\
\hline 8-Bromo-1-octanol & $0 \cdot 8559$ & $0 \cdot 4490$ & 8 & 2.79 & $1 \cdot 0424$ & 0.5921 & 0.7848 \\
\hline 2,3-Dibromopropanol & 0.9902 & 0.4599 & 3 & $0 \cdot 63$ & $-0 \cdot 4861$ & $-1 \cdot 0434$ & $-1 \cdot 0507$ \\
\hline \multicolumn{8}{|l|}{ Saturated alcohol } \\
\hline Methyl alcohol & $0 \cdot 9485$ & $0 \cdot 4440$ & 1 & -0.77 & $-2 \cdot 6656$ & -2.6657 & $-2 \cdot 6755$ \\
\hline Ethyl alcohol & $0 \cdot 9186$ & $0 \cdot 4481$ & 2 & $-0 \cdot 31$ & -1.9912 & $-2 \cdot 2513$ & $-2 \cdot 2761$ \\
\hline 1-Propanol & $0 \cdot 8979$ & $0 \cdot 4485$ & 3 & $0 \cdot 25$ & $-1 \cdot 7464$ & $-1 \cdot 8369$ & $-1 \cdot 8685$ \\
\hline 2-Propanol & 0.9500 & $0 \cdot 4548$ & 3 & 0.05 & $-1 \cdot 8819$ & -1.8369 & $-1 \cdot 822$ \\
\hline 1-Butanol & $0 \cdot 8960$ & $0 \cdot 4484$ & 4 & 0.88 & -1.4306 & $-1 \cdot 4225$ & $-1 \cdot 4441$ \\
\hline$(+/-)-2-$ Butanol & $0 \cdot 9227$ & $0 \cdot 4480$ & 4 & $0 \cdot 61$ & $-1 \cdot 542$ & $-1 \cdot 4225$ & $-1 \cdot 4202$ \\
\hline 2-Methyl-1-propanol & $0 \cdot 9066$ & $0 \cdot 4501$ & 4 & $0 \cdot 76$ & $-1 \cdot 3724$ & $-1 \cdot 4225$ & $-1 \cdot 4346$ \\
\hline 2-Pentanol & $0 \cdot 9045$ & $0 \cdot 4479$ & 5 & $1 \cdot 19$ & $-1 \cdot 1596$ & $-1 \cdot 0081$ & $-1 \cdot 0104$ \\
\hline 3-Pentanol & $0 \cdot 8945$ & 0.4569 & 5 & $1 \cdot 21$ & $-1 \cdot 2437$ & $-1 \cdot 0081$ & $-1 \cdot 0193$ \\
\hline 3-Methyl-2-butanol & 0.8935 & 0.4482 & 5 & $1 \cdot 28$ & -0.9959 & $-1 \cdot 0081$ & $-1 \cdot 0202$ \\
\hline tert-amylalcohol & 0.9354 & 0.4459 & 5 & 0.89 & $-1 \cdot 1729$ & $-1 \cdot 0081$ & $-0 \cdot 9828$ \\
\hline 2-Methyl-1-butanol & $0 \cdot 9034$ & $0 \cdot 4502$ & 5 & $1 \cdot 22$ & -0.9528 & $-1 \cdot 0081$ & $-1 \cdot 0114$ \\
\hline 3-Methyl-1-butanol & $0 \cdot 9218$ & $0 \cdot 4481$ & 5 & $1 \cdot 16$ & -1.0359 & $-1 \cdot 0081$ & -0.9949 \\
\hline 2,2-Dimethyl-1-propanol & $0 \cdot 9416$ & $0 \cdot 4516$ & 4 & $1 \cdot 31$ & $-0 \cdot 8702$ & $-1 \cdot 4225$ & $-1 \cdot 4034$ \\
\hline 2-Methyl-2-propanol & 0.9560 & $0 \cdot 4446$ & 4 & $0 \cdot 35$ & $-1 \cdot 7911$ & $-1 \cdot 4225$ & $-1 \cdot 3905$ \\
\hline 1-Hexanol & $0 \cdot 8955$ & $0 \cdot 4484$ & 6 & $2 \cdot 03$ & -0.3789 & -0.5936 & -0.5923 \\
\hline 3,3-Dimethyl-1-butanol & $0 \cdot 9357$ & $0 \cdot 4483$ & 5 & $1 \cdot 62$ & -0.7368 & $-1 \cdot 0081$ & -0.9825 \\
\hline 4-Methyl-1-pentanol & 0.9354 & $0 \cdot 4484$ & 6 & 1.75 & -0.6372 & $-0 \cdot 5936$ & -0.5567 \\
\hline 1-Heptanol & $0 \cdot 8958$ & $0 \cdot 4484$ & 7 & $2 \cdot 72$ & $0 \cdot 1050$ & $-0 \cdot 1792$ & $-0 \cdot 1659$ \\
\hline 2,4-Dimethyl-3-pentanol & $0 \cdot 8519$ & $0 \cdot 4525$ & 7 & 1.93 & $-0 \cdot 7052$ & $-0 \cdot 1792$ & $-0 \cdot 2051$ \\
\hline 1-Octanol & $0 \cdot 8769$ & $0 \cdot 4483$ & 8 & $3 \cdot 00$ & $0 \cdot 5827$ & $0 \cdot 2352$ & $0 \cdot 2433$ \\
\hline 2-Octanol & $0 \cdot 8779$ & $0 \cdot 4479$ & 8 & $2 \cdot 90$ & $0 \cdot 0011$ & $0 \cdot 2352$ & $0 \cdot 2442$ \\
\hline 3-Octanol & $0 \cdot 8560$ & $0 \cdot 4511$ & 8 & $2 \cdot 72$ & $0 \cdot 0309$ & $0 \cdot 2352$ & $0 \cdot 2247$ \\
\hline 1-Nonanol & $0 \cdot 8560$ & $0 \cdot 4483$ & 9 & $3 \cdot 77$ & $0 \cdot 8551$ & $0 \cdot 6496$ & $0 \cdot 6508$ \\
\hline 2-Nonanol & $0 \cdot 8658$ & $0 \cdot 4479$ & 9 & $3 \cdot 25$ & $0 \cdot 6183$ & $0 \cdot 6496$ & $0 \cdot 6595$ \\
\hline 3-Ethyl-2,2-dimethyl-3-pentanol & $0 \cdot 8221$ & 0.4483 & 9 & $2 \cdot 86$ & $-0 \cdot 1691$ & $0 \cdot 6496$ & 0.6205 \\
\hline
\end{tabular}


Table 1. (Contd...)

\begin{tabular}{|c|c|c|c|c|c|c|c|}
\hline \multirow[b]{2}{*}{ Molecules } & \multirow[b]{2}{*}{$\omega$} & \multirow[b]{2}{*}{$Q_{k}^{\max }$} & \multirow[b]{2}{*}{$N c$} & \multirow[b]{2}{*}{$\log P^{*}$} & \multicolumn{3}{|c|}{$\mathrm{pIGC}_{50}$} \\
\hline & & & & & Experiment* & Calc. $(N c)$ & Calc. $(N c, \omega)$ \\
\hline 1-Decanol & $0 \cdot 8387$ & 0.4483 & 10 & $4 \cdot 57$ & $1 \cdot 3354$ & $1 \cdot 0640$ & $1 \cdot 0614$ \\
\hline$(+/-)-4-$ Decanol & $0 \cdot 8182$ & 0.4512 & 10 & $3 \cdot 78$ & $0 \cdot 8499$ & $1 \cdot 0640$ & $1 \cdot 0431$ \\
\hline 3,7-Dimethyl-3-octanol & $0 \cdot 8658$ & 0.4450 & 10 & $3 \cdot 52$ & $0 \cdot 3404$ & $1 \cdot 0640$ & $1 \cdot 0856$ \\
\hline 1-Undecanol & $0 \cdot 8248$ & $0 \cdot 4483$ & 10 & $4 \cdot 53$ & 1.9547 & $1 \cdot 0640$ & $1 \cdot 049$ \\
\hline 1-Dodecanol & $0 \cdot 8132$ & $0 \cdot 4483$ & 12 & $5 \cdot 13$ & $2 \cdot 1612$ & $1 \cdot 8928$ & 1.8909 \\
\hline 1-Tridecanol & $0 \cdot 8035$ & 0.4483 & 13 & $5 \cdot 58$ & $2 \cdot 4497$ & $2 \cdot 3072$ & $2 \cdot 3083$ \\
\hline \multicolumn{8}{|l|}{ Carboxylic acid } \\
\hline Propionic acid & 0.9901 & $0 \cdot 9780$ & 3 & $0 \cdot 33$ & $-0 \cdot 5123$ & -0.6331 & $-0 \cdot 431$ \\
\hline Butyric acid & $1 \cdot 0051$ & $0 \cdot 9840$ & 4 & 0.79 & -0.5720 & -0.5216 & -0.4788 \\
\hline Valeric acid & $0 \cdot 9840$ & $0 \cdot 9851$ & 5 & $1 \cdot 39$ & $-0 \cdot 2674$ & $-0 \cdot 4100$ & $-0 \cdot 3301$ \\
\hline Hexanoic acid & 0.9731 & $0 \cdot 9852$ & 6 & 1.92 & $-0 \cdot 2083$ & $-0 \cdot 2984$ & $-0 \cdot 2369$ \\
\hline Heptanoic acid & $0 \cdot 9582$ & 0.9853 & 7 & $2 \cdot 41$ & $-0 \cdot 1126$ & $-0 \cdot 1868$ & $-0 \cdot 122$ \\
\hline Octanoic acid & 0.9397 & $0 \cdot 9852$ & 8 & $3 \cdot 05$ & $0 \cdot 0807$ & $-0 \cdot 0753$ & $0 \cdot 0126$ \\
\hline Nonanoic acid & 0.9184 & $0 \cdot 9853$ & 9 & $3 \cdot 47$ & $0 \cdot 3509$ & 0.0363 & $0 \cdot 1623$ \\
\hline Decanoic acid & $0 \cdot 8986$ & 0.9853 & 10 & $4 \cdot 09$ & $0 \cdot 5063$ & $0 \cdot 1478$ & $0 \cdot 3039$ \\
\hline Undecanoic acid & $0 \cdot 8813$ & $0 \cdot 9853$ & 11 & $4 \cdot 53$ & $0 \cdot 8983$ & $0 \cdot 2594$ & 0.4319 \\
\hline iso-Butyric acid & $0 \cdot 9624$ & 0.9834 & 4 & $0 \cdot 60$ & $-0 \cdot 3334$ & $-0 \cdot 5216$ & $-0 \cdot 2464$ \\
\hline Isovalerianic acid & $1 \cdot 0071$ & $0 \cdot 9823$ & 5 & $1 \cdot 16$ & $-0 \cdot 3415$ & $-0 \cdot 4100$ & -0.4558 \\
\hline Trimethylacetic acid & 0.9574 & $0 \cdot 9819$ & 5 & 1.47 & $-0 \cdot 2543$ & $-0 \cdot 4100$ & $-0 \cdot 1853$ \\
\hline 3-Methylvaleric acid & 0.9657 & $0 \cdot 9884$ & 6 & $1 \cdot 75$ & $-0 \cdot 2331$ & $-0 \cdot 2984$ & $-0 \cdot 1966$ \\
\hline 4-Methylvaleric acid & 0.9964 & $0 \cdot 9871$ & 6 & $1 \cdot 75$ & $-0 \cdot 2724$ & $-0 \cdot 2984$ & $-0 \cdot 3637$ \\
\hline 2-Ethylbutyric acid & 0.9355 & $0 \cdot 9854$ & 6 & $1 \cdot 68$ & $-0 \cdot 1523$ & $-0 \cdot 2984$ & -0.0323 \\
\hline 2-Propylpentanoic acid & $0 \cdot 8905$ & $0 \cdot 9903$ & 8 & $2 \cdot 75$ & $0 \cdot 0258$ & $-0 \cdot 0753$ & $0 \cdot 2803$ \\
\hline 2-Ethylhexanoic acid & $0 \cdot 9122$ & 0.9896 & 8 & $2 \cdot 64$ & $0 \cdot 0756$ & -0.0753 & $0 \cdot 1622$ \\
\hline Succinic acid & $1 \cdot 0511$ & 0.9829 & 4 & $-0 \cdot 59$ & -0.9395 & $-0 \cdot 5216$ & -0.7291 \\
\hline Glutaric acid & $1 \cdot 0756$ & 0.9839 & 5 & $-0 \cdot 29$ & -0.6387 & $-0 \cdot 4100$ & -0.8286 \\
\hline Adipic acid & $1 \cdot 0345$ & $0 \cdot 9850$ & 6 & $0 \cdot 08$ & -0.606 & $-0 \cdot 2984$ & $-0 \cdot 5711$ \\
\hline Pimelic acid & $1 \cdot 0336$ & $0 \cdot 9848$ & 7 & $0 \cdot 42$ & $-0 \cdot 5845$ & $-0 \cdot 1868$ & -0.5323 \\
\hline 3,3-Dimethylglutaric acid & $1 \cdot 0614$ & $0 \cdot 9856$ & 7 & $0 \cdot 16$ & $-0 \cdot 6643$ & $-0 \cdot 1868$ & -0.6837 \\
\hline Suberic acid & 0.9991 & $0 \cdot 9852$ & 8 & $0 \cdot 95$ & $-0 \cdot 5116$ & $-0 \cdot 0753$ & $-0 \cdot 3107$ \\
\hline Sebacic acid & 0.9600 & $0 \cdot 9853$ & 10 & $2 \cdot 01$ & $-0 \cdot 2676$ & $0 \cdot 1478$ & -0.0302 \\
\hline 1,10-Decanedicarboxylic acid & $0 \cdot 9181$ & $0 \cdot 9853$ & 12 & $3 \cdot 07$ & -0.0863 & $0 \cdot 3710$ & $0 \cdot 2655$ \\
\hline Crotonic acid & $1 \cdot 0041$ & $0 \cdot 9462$ & 4 & $0 \cdot 72$ & $-0 \cdot 5448$ & $-0 \cdot 5216$ & $-0 \cdot 4733$ \\
\hline trans-2-Pentenoic acid & $1 \cdot 0254$ & $0 \cdot 9496$ & 5 & $1 \cdot 41$ & $-0 \cdot 2774$ & $-0 \cdot 4100$ & -0.5554 \\
\hline trans-2-Hexenoic acid & 0.9961 & 0.9469 & 5 & $1 \cdot 94$ & $-0 \cdot 1279$ & $-0 \cdot 4100$ & $-0 \cdot 3959$ \\
\hline \multicolumn{8}{|l|}{ Halogenated acid } \\
\hline 4-Bromobutyric acid & $0 \cdot 6742$ & $0 \cdot 9994$ & 4 & $0 \cdot 68$ & -0.7711 & $-0 \cdot 4453$ & -0.6158 \\
\hline 5-Bromovaleric acid & $0 \cdot 6476$ & 0.9992 & 5 & $1 \cdot 21$ & -0.6929 & $-0 \cdot 2197$ & $-0 \cdot 5685$ \\
\hline 4-Chlorobutyric acid & 0.6786 & 0.9994 & 4 & $0 \cdot 54$ & -0.6773 & -0.4453 & -0.6075 \\
\hline 3-Chloropropionic acid & 0.7333 & 0.9952 & 3 & 0.41 & $-0 \cdot 3321$ & $-0 \cdot 6710$ & $-0 \cdot 6016$ \\
\hline 5-Chlorovaleric acid & $0 \cdot 6419$ & 0.9992 & 5 & $1 \cdot 07$ & $-0 \cdot 2857$ & $-0 \cdot 2197$ & -0.5793 \\
\hline 2-Bromobutyric acid & $1 \cdot 0508$ & $0 \cdot 9800$ & 4 & 1.42 & $0 \cdot 1221$ & -0.4453 & 0.0971 \\
\hline 2-Bromoisobutyric acid & $0 \cdot 7178$ & 0.9825 & 4 & $0 \cdot 86$ & $-0 \cdot 5845$ & $-0 \cdot 4453$ & -0.5333 \\
\hline 2-Bromoisovaleric acid & 0.7562 & $0 \cdot 9826$ & 5 & 1.48 & $-0 \cdot 5492$ & $-0 \cdot 2197$ & $-0 \cdot 3629$ \\
\hline 2-Bromovaleric acid & $1 \cdot 0422$ & 0.9806 & 5 & $1 \cdot 61$ & -0.0423 & -0.2197 & $0 \cdot 1785$ \\
\hline 2-Bromooctanoic acid & $1 \cdot 0345$ & 0.9806 & 8 & $3 \cdot 19$ & $0 \cdot 4907$ & $0 \cdot 4574$ & 0.4569 \\
\hline 2-Bromohexanoic acid & $1 \cdot 0382$ & 0.9806 & 6 & $2 \cdot 14$ & 0.4547 & $0 \cdot 0060$ & $0 \cdot 2686$ \\
\hline \multicolumn{8}{|l|}{ Mono ester } \\
\hline Ethyl acetate & $0 \cdot 9420$ & 0.9792 & 4 & $0 \cdot 73$ & $-1 \cdot 2968$ & $-1 \cdot 3388$ & $-1 \cdot 1201$ \\
\hline Propyl acetate & $0 \cdot 9562$ & 0.9799 & 5 & $1 \cdot 24$ & $-1 \cdot 2382$ & -0.9743 & $-1 \cdot 0196$ \\
\hline Isopropyl acetate & 0.9664 & 0.9826 & 5 & 1.02 & $-1 \cdot 5900$ & -0.9743 & $-1 \cdot 1309$ \\
\hline Butyl acetate & 0.9465 & $0 \cdot 9801$ & 6 & $1 \cdot 78$ & $-0 \cdot 4864$ & -0.6098 & -0.6583 \\
\hline
\end{tabular}


Table 1. (Contd...)

\begin{tabular}{|c|c|c|c|c|c|c|c|}
\hline \multirow[b]{2}{*}{ Molecules } & \multirow[b]{2}{*}{$\omega$} & \multirow[b]{2}{*}{$Q_{k}^{\max }$} & \multirow[b]{2}{*}{$N c$} & \multirow[b]{2}{*}{$\log P^{*}$} & \multicolumn{3}{|c|}{$\mathrm{pIGC}_{50}$} \\
\hline & & & & & Experiment* & Calc. $(N C)$ & Calc. $(N c, \omega)$ \\
\hline Amyl acetate & $0 \cdot 9408$ & $0 \cdot 9801$ & 7 & $2 \cdot 30$ & $0 \cdot 1625$ & $-0 \cdot 2453$ & $-0 \cdot 3407$ \\
\hline Hexyl acetate & 0.9328 & 0.9801 & 8 & $2 \cdot 83$ & -0.0087 & $0 \cdot 1192$ & $0 \cdot 002$ \\
\hline Octyl acetate & 0.9115 & $0 \cdot 9801$ & 11 & $3 \cdot 88$ & 1.0570 & $1 \cdot 2128$ & $1 \cdot 0007$ \\
\hline Decyl acetate & $0 \cdot 8803$ & 0.9801 & 12 & $4 \cdot 94$ & $1 \cdot 8794$ & 1.5773 & 1.5966 \\
\hline Ethyl propionate & 0.9443 & 0.9821 & 5 & $1 \cdot 21$ & -0.9450 & -0.9743 & $-0 \cdot 8897$ \\
\hline Butyl propionate & 0.9379 & 0.9829 & 7 & $2 \cdot 30$ & $0 \cdot 1704$ & $-0 \cdot 2453$ & $-0 \cdot 3091$ \\
\hline Isobutyl propionate & 0.9721 & 0.9837 & 7 & $2 \cdot 17$ & -0.6935 & $-0 \cdot 2453$ & $-0 \cdot 6823$ \\
\hline Propyl propionate & 0.9502 & 0.9827 & 6 & $1 \cdot 77$ & -0.8148 & -0.6098 & -0.6987 \\
\hline tert-Butyl propionate & 0.9288 & 0.9847 & 7 & $1 \cdot 95$ & -0.4095 & $-0 \cdot 2453$ & $-0 \cdot 2098$ \\
\hline Ethyl butyrate & 0.9486 & 0.9879 & 6 & $1 \cdot 77$ & -0.4903 & -0.6098 & -0.6813 \\
\hline Ethyl isobutyrate & 0.9406 & 0.9843 & 6 & $1 \cdot 55$ & $-1 \cdot 2709$ & -0.6098 & -0.594 \\
\hline Ethyl valerate & 0.9346 & 0.9889 & 7 & $2 \cdot 30$ & $-0 \cdot 3580$ & $-0 \cdot 2453$ & $-0 \cdot 2731$ \\
\hline Propyl butyrate & 0.9483 & 0.9886 & 7 & $2 \cdot 30$ & -0.4138 & $-0 \cdot 2453$ & -0.4226 \\
\hline Butyl butyrate & 0.9374 & 0.9887 & 8 & $2 \cdot 83$ & 0.5157 & $0 \cdot 1192$ & $-0 \cdot 0482$ \\
\hline Propyl valerate & 0.9381 & 0.9896 & 8 & $2 \cdot 83$ & 0.0094 & $0 \cdot 1192$ & $-0 \cdot 0558$ \\
\hline Amyl propionate & 0.9317 & 0.9829 & 5 & $2 \cdot 83$ & -0.0431 & -0.9743 & -0.7522 \\
\hline Ethyl hexanoate & 0.9248 & 0.9891 & 6 & $2 \cdot 83$ & 0.0637 & $-0 \cdot 6098$ & -0.4215 \\
\hline Methyl butyrate & 0.9518 & 0.9832 & 5 & $1 \cdot 29$ & $-1 \cdot 2463$ & -0.9743 & -0.9716 \\
\hline Methyl valerate & 0.9380 & 0.9843 & 6 & $1 \cdot 96$ & $-0 \cdot 8448$ & -0.6098 & -0.5656 \\
\hline Methyl hexanoate & 0.9271 & 0.9845 & 7 & $2 \cdot 30$ & $-0 \cdot 5611$ & $-0 \cdot 2453$ & $-0 \cdot 1912$ \\
\hline Methyl heptanoate & 0.9157 & 0.9844 & 8 & $2 \cdot 83$ & $0 \cdot 1039$ & $0 \cdot 1192$ & $0 \cdot 1886$ \\
\hline Methyl octanoate & 0.9027 & 0.9845 & 9 & $3 \cdot 36$ & 0.5358 & 0.4837 & 0.5859 \\
\hline Methyl nonanoate & $0 \cdot 8868$ & 0.9845 & 10 & $3 \cdot 88$ & $1 \cdot 0419$ & $0 \cdot 8482$ & 1.0149 \\
\hline Methyl decanoate & $0 \cdot 8710$ & 0.9845 & 11 & $4 \cdot 41$ & $1 \cdot 3778$ & $1 \cdot 2128$ & 1.4427 \\
\hline Methyl undecanoate & $0 \cdot 8562$ & 0.9845 & 12 & $4 \cdot 79$ & 1.4248 & 1.5773 & 1.8596 \\
\hline Methyl formate & 0.9611 & 0.8265 & 2 & $0 \cdot 03$ & -1.4982 & -2.0679 & -1.8393 \\
\hline tert-Butyl formate & 0.9594 & 0.8379 & 5 & 0.97 & $-1 \cdot 3719$ & -0.9743 & $-1 \cdot 0545$ \\
\hline \multicolumn{8}{|l|}{ Di ester } \\
\hline Diethyl malonate & $0 \cdot 6983$ & $1 \cdot 0038$ & 7 & $0 \cdot 96$ & -0.9975 & $-0 \cdot 8809$ & $-0 \cdot 8413$ \\
\hline Diethyl sebacate & $0 \cdot 5494$ & 1.0009 & 14 & $3 \cdot 90$ & $1 \cdot 3536$ & $1 \cdot 1221$ & $1 \cdot 2753$ \\
\hline Diethyl suberate & 0.5705 & $1 \cdot 0010$ & 12 & $2 \cdot 84$ & 0.7018 & $0 \cdot 5498$ & 0.7738 \\
\hline Diethyl succinate & 0.6764 & 0.9996 & 8 & $1 \cdot 19$ & $-0 \cdot 8511$ & $-0 \cdot 5948$ & -0.5359 \\
\hline Dimethyl malonate & 0.7367 & 0.9994 & 5 & -0.05 & $-1 \cdot 2869$ & -1.4532 & $-1 \cdot 4261$ \\
\hline Dibutyl adipate & 0.5838 & $1 \cdot 0017$ & 14 & 3.90 & 0.7918 & $1 \cdot 1221$ & $1 \cdot 1096$ \\
\hline Dimethyl succinate & 0.7085 & 0.9953 & 6 & $0 \cdot 35$ & -1.0573 & $-1 \cdot 1671$ & -1.0904 \\
\hline Diethyl adipate & 0.5991 & 1.0010 & 10 & $1 \cdot 79$ & $-0 \cdot 1265$ & $-0 \cdot 0225$ & $0 \cdot 2362$ \\
\hline Dimethyl brassylate & $0 \cdot 5361$ & 0.9966 & 15 & $4 \cdot 43$ & 1.6536 & 1.4083 & 1.5392 \\
\hline Dimethyl sebacate & 0.5703 & 0.9967 & 12 & $2 \cdot 84$ & $1 \cdot 0106$ & $0 \cdot 5498$ & 0.7748 \\
\hline Dimethyl suberate & 0.5952 & 0.9967 & 10 & $1 \cdot 79$ & $0 \cdot 2962$ & -0.0225 & $0 \cdot 255$ \\
\hline Diethyl pimelate & 0.5759 & 1.0006 & 11 & $2 \cdot 31$ & 0.4069 & $0 \cdot 2636$ & 0.5479 \\
\hline Dibutyl suberate & 0.5574 & $1 \cdot 0017$ & 16 & $4 \cdot 96$ & $1 \cdot 6556$ & 1.6944 & $1 \cdot 6366$ \\
\hline Diethyl butylmalonate & 0.6795 & $1 \cdot 0140$ & 11 & $3 \cdot 02$ & 0.5566 & $0 \cdot 2636$ & 0.0489 \\
\hline Diethyl ethylmalonate & 0.6916 & $1 \cdot 0133$ & 9 & $1 \cdot 96$ & $-0 \cdot 2422$ & $-0 \cdot 3086$ & $-0 \cdot 4092$ \\
\hline Diethyl-3-oxopimelate & 0.7225 & 1.0050 & 11 & 1.49 & $-0 \cdot 3778$ & 0.2636 & $-0 \cdot 1582$ \\
\hline Diethyl-4-oxopimelate & 0.7458 & 1.0002 & 11 & $1 \cdot 54$ & -0.6378 & $0 \cdot 2636$ & $-0 \cdot 2705$ \\
\hline Diethyl methylmalonate & 0.7004 & 1.0079 & 8 & $1 \cdot 44$ & $-0 \cdot 5114$ & -0.5948 & -0.6515 \\
\hline Diethyl propylmalonate & $0 \cdot 6837$ & $1 \cdot 0140$ & 10 & $2 \cdot 49$ & $0 \cdot 1341$ & $-0 \cdot 0225$ & $-0 \cdot 1713$ \\
\hline Dibutyl succinate & $0 \cdot 6602$ & 1.0003 & 12 & $3 \cdot 60$ & 0.5123 & 0.5498 & $0 \cdot 3418$ \\
\hline \multicolumn{8}{|l|}{ Aldehyde } \\
\hline Propionaldehyde & $0 \cdot 8905$ & 0.5592 & 3 & 0.59 & -0.4855 & -0.7336 & -0.5798 \\
\hline Butyraldehyde & $0 \cdot 8722$ & 0.5638 & 4 & $0 \cdot 88$ & $-0 \cdot 3805$ & $-0 \cdot 5106$ & -0.4108 \\
\hline Isobutyraldehyde & 0.9295 & 0.5589 & 4 & $0 \cdot 61$ & -0.4328 & -0.5106 & $-0 \cdot 5555$ \\
\hline
\end{tabular}


Table 1. (Contd...)

\begin{tabular}{|c|c|c|c|c|c|c|c|}
\hline \multirow[b]{2}{*}{ Molecules } & \multirow[b]{2}{*}{$\omega$} & \multirow[b]{2}{*}{$Q_{k}^{\max }$} & \multirow[b]{2}{*}{$N c$} & \multirow[b]{2}{*}{$\log P^{*}$} & \multicolumn{3}{|c|}{$\mathrm{pIGC}_{50}$} \\
\hline & & & & & Experiment* & Calc. $(N c)$ & Calc. $(N c, \omega)$ \\
\hline Valeraldehyde & $0 \cdot 8620$ & $0 \cdot 5651$ & 5 & $1 \cdot 36$ & $-0 \cdot 0223$ & $-0 \cdot 2876$ & $-0 \cdot 2623$ \\
\hline 2-Methyl-butyraldehyde & $0 \cdot 8494$ & $0 \cdot 5644$ & 5 & $1 \cdot 14$ & $-0 \cdot 3107$ & $-0 \cdot 2876$ & $-0 \cdot 2305$ \\
\hline Hexylaldehyde & $0 \cdot 8384$ & $0 \cdot 5624$ & 6 & 1.78 & $-0 \cdot 1731$ & -0.0646 & -0.0799 \\
\hline 2-Methylvaleraldehyde & $0 \cdot 8354$ & $0 \cdot 5655$ & 6 & $1 \cdot 67$ & $-0 \cdot 4745$ & $-0 \cdot 0646$ & -0.0723 \\
\hline 2-Ethylbutyraldehyde & $0 \cdot 8429$ & $0 \cdot 5542$ & 6 & $1 \cdot 67$ & $-0 \cdot 0544$ & -0.0646 & -0.0913 \\
\hline 3,3-Dimethylbutyraldehyde & 0.9114 & $0 \cdot 5579$ & 6 & 1.63 & $-0 \cdot 3744$ & $-0 \cdot 0646$ & $-0 \cdot 2642$ \\
\hline Heptaldehyde & $0 \cdot 8517$ & 0.5653 & 7 & $2 \cdot 42$ & $-0 \cdot 0019$ & $0 \cdot 1584$ & $0 \cdot 0093$ \\
\hline 2-Ethylhexanal & $0 \cdot 8268$ & 0.5555 & 8 & 2.73 & $0 \cdot 1608$ & $0 \cdot 3814$ & $0 \cdot 1949$ \\
\hline trans-4-Decen-1-al & 0.6717 & $0 \cdot 5642$ & 10 & $4 \cdot 05$ & $1 \cdot 2076$ & $0 \cdot 8275$ & $0 \cdot 832$ \\
\hline cis-7-Decen-1-al & 0.5588 & 0.5652 & 10 & $3 \cdot 52$ & 0.9485 & $0 \cdot 8275$ & $1 \cdot 1171$ \\
\hline \multicolumn{8}{|l|}{ Ketones } \\
\hline Acetone & $0 \cdot 8709$ & 0.6969 & 3 & $-0 \cdot 24$ & $-2 \cdot 2036$ & $-2 \cdot 203$ & $-2 \cdot 2784$ \\
\hline 2-Butanone & $0 \cdot 8544$ & $0 \cdot 7020$ & 4 & 0.29 & $-1 \cdot 7457$ & $-1 \cdot 7884$ & $-1 \cdot 8354$ \\
\hline 2-Pentanone & $0 \cdot 8175$ & 0.7072 & 5 & 0.91 & $-1 \cdot 2224$ & $-1 \cdot 3737$ & $-1 \cdot 3268$ \\
\hline 3-Pentanone & $0 \cdot 8315$ & 0.7033 & 5 & 0.85 & -1.4561 & $-1 \cdot 3737$ & $-1 \cdot 3719$ \\
\hline 4-Methyl-2-pentanone & $0 \cdot 8315$ & $0 \cdot 7060$ & 6 & $1 \cdot 31$ & $-1 \cdot 2085$ & -0.9590 & -0.982 \\
\hline 2-Heptanone & 0.7975 & 0.7084 & 7 & 1.98 & -0.4872 & $-0 \cdot 5444$ & -0.4827 \\
\hline 5-Methyl-2-hexanone & $0 \cdot 8053$ & $0 \cdot 7105$ & 7 & $1 \cdot 88$ & -0.6459 & -0.5444 & -0.5078 \\
\hline 4-Heptanone & $0 \cdot 8108$ & $0 \cdot 7151$ & 7 & $1 \cdot 91$ & $-0 \cdot 6690$ & -0.5444 & -0.5255 \\
\hline 2-Octanone & 0.7948 & $0 \cdot 7085$ & 8 & $2 \cdot 37$ & $-0 \cdot 1455$ & $-0 \cdot 1297$ & -0.0841 \\
\hline 2-Nonanone & 0.7926 & 0.7085 & 9 & $3 \cdot 14$ & 0.6598 & $0 \cdot 2849$ & $0 \cdot 3129$ \\
\hline 2-Decanone & $0 \cdot 7912$ & 0.7085 & 10 & $3 \cdot 73$ & $0 \cdot 5822$ & $0 \cdot 6996$ & 0.7072 \\
\hline 3-Decanone & 0.7992 & 0.7171 & 10 & $3 \cdot 49$ & 0.6265 & 0.6996 & 0.6815 \\
\hline 2-Undecanone & 0.7901 & $0 \cdot 7085$ & 11 & 4.09 & 1.5346 & $1 \cdot 1142$ & $1 \cdot 1007$ \\
\hline 2-Dodecanone & 0.7893 & $0 \cdot 7085$ & 12 & $4 \cdot 55$ & 1.6696 & $1 \cdot 5289$ & 1.4931 \\
\hline 7-Tridecanone & $0 \cdot 7811$ & 0.7177 & 13 & $5 \cdot 08$ & $1 \cdot 5214$ & 1.9435 & 1.9094 \\
\hline
\end{tabular}

*Taken from reference 10

as expected. Except for the set of aliphatic amines the atom number is a valuable descriptor. The global electrophilicity is also a reliable descriptor in most cases. It is found that on an average the twoparameter models with the use of number of carbon atom $\left(N_{\mathrm{C}}\right)$ and the electrophilicity index $(\omega)$ provide almost equivalent prediction compared to the corresponding three-parameter models by adding one extra descriptor from the rest $\left(N_{\mathrm{NH}}, \omega_{\max }^{+}, \omega_{\max }^{-}\right.$and $\left.Q_{k}^{\max }\right)$. Therefore, we report only the one- and two-parameter results obtained using $N_{C}$ and $\omega$ except for the amines in which an extra potent descriptor $Q_{k}^{\max }$ is included.

Experimental and calculated $\mathrm{pIGC}_{50}$ values along with various descriptors are presented in tables 1 and 2 for the electron acceptor and electron donor molecules respectively. Table 3 presents the corresponding regression equations associated with one $\left(N_{C}\right)$ - and two $\left(N_{\mathrm{C}}, \omega\right)$-parameter models. The single parameter model with a simple descriptor like the number of carbon atoms provides good estimates of toxicity in most cases. The exceptions are carboxylic acids, halogenated acids, amino alcohols and amines. Situation improves drastically in all cases by including $\omega$ with the $N_{\mathrm{C}}$ except for amines where the information of charge is also important. It is important to note that $N_{\mathrm{C}}$ may be considered to be a crude alternative to $\log P$ (table 3 ) in developing QSTR model which can be further improved by including $\omega$ and/or its local counterpart as well as charges on the reactive centers. In certain cases $N_{\mathrm{C}}$ and $\log P$ (along with other descriptors mentioned above) provide comparable results. The constant terms in the twoparameter models are not always significant. Figure 1 presents the plots of experimental $\mathrm{pIGC}_{50}$ versus calculated $\mathrm{pIGC}_{50}$ values for the (a) acceptor set $\left(R^{2}=0.9283, R_{\mathrm{cv}}^{2}=0.9265, R_{\mathrm{adj}}^{2}=0.9279\right)$ and $(\mathrm{b})$ donor set $\left(R^{2}=0.8284, R_{\mathrm{cv}}^{2}=0.8156, R_{\mathrm{adj}}^{2}=0.8262\right)$ which authenticates the efficacy of these regression models for QSTR. It is also important to note that the slopes of these plots are unity and the intercepts are very close to zero, as expected. Although the pre- 
Table 2. Electrophilicity $(\omega)$, maximum atomic charge $\left(Q_{k}^{\max }\right)$, number of carbon atoms $(N c)$, $\log P$ along with the experimental and calculated values of $\log \left(\mathrm{IGC}_{50}{ }^{-1}\right)$ for the complete set of aliphatic donor compounds with Tetrahymena pyriformis.

\begin{tabular}{|c|c|c|c|c|c|c|c|}
\hline \multirow[b]{2}{*}{ Molecules } & \multirow[b]{2}{*}{$\omega$} & \multirow[b]{2}{*}{$Q_{k}^{\max }$} & \multirow[b]{2}{*}{$N c$} & \multirow[b]{2}{*}{$\log P^{*}$} & \multicolumn{3}{|c|}{$\mathrm{pIGC}_{50}$} \\
\hline & & & & & Experiment* & Calc. $(N c)$ & Calc. $(N c, \omega)$ \\
\hline \multicolumn{8}{|l|}{ Amino alcohol } \\
\hline 2-(Methylamino)ethanol & $0 \cdot 5611$ & -0.7684 & 3 & $-0 \cdot 94$ & $-1 \cdot 8202$ & $-1 \cdot 6530$ & $-1 \cdot 961$ \\
\hline 4-Amino-1-butanol & $0 \cdot 6562$ & -0.8278 & 4 & $-1 \cdot 06$ & -0.9752 & $-1 \cdot 4275$ & -0.9598 \\
\hline 2-(Ethylamino)ethanol & $0 \cdot 5658$ & -0.7656 & 4 & $-0 \cdot 46$ & $-1 \cdot 6491$ & $-1 \cdot 4275$ & $-1 \cdot 7723$ \\
\hline 2-Propylaminoethanol & $0 \cdot 5548$ & -0.7657 & 5 & $0 \cdot 07$ & $-1 \cdot 6842$ & $-1 \cdot 2020$ & $-1 \cdot 7248$ \\
\hline DL-2-amino-1-pentanol & 0.6623 & -0.8457 & 5 & $0 \cdot 07$ & $-0 \cdot 6718$ & $-1 \cdot 2020$ & -0.7586 \\
\hline 3-Amino-2,2-dimethyl-1-propanol & $0 \cdot 6792$ & $-0 \cdot 8558$ & 5 & -0.79 & -0.9246 & $-1 \cdot 2020$ & -0.6067 \\
\hline 6-Amino-1-hexanol & 0.6297 & -0.8512 & 6 & $-0 \cdot 01$ & -0.958 & -0.9764 & -0.9052 \\
\hline DL-2-amino-1-hexanol & $0 \cdot 6621$ & $-0 \cdot 8458$ & 6 & $0 \cdot 60$ & $-0 \cdot 5848$ & $-0 \cdot 9764$ & $-0 \cdot 614$ \\
\hline DL-2-amino-3-methyl-1-butanol & 0.6306 & $-0 \cdot 8569$ & 5 & $-0 \cdot 06$ & $-0 \cdot 5852$ & $-1 \cdot 2020$ & $-1 \cdot 0435$ \\
\hline 2-Amino-3,3-dimethyl-butanol & $0 \cdot 6430$ & $-0 \cdot 8599$ & 6 & $0 \cdot 34$ & -0.7178 & $-0 \cdot 9764$ & -0.7857 \\
\hline 2-Amino-3-methyl-1-pentanol & 0.6325 & -0.8607 & 6 & $0 \cdot 47$ & -0.6594 & -0.9764 & $-0 \cdot 88$ \\
\hline 2-Amino-4-methyl-pentanol & $0 \cdot 6484$ & -0.8574 & 6 & 0.47 & $-0 \cdot 6191$ & -0.9764 & -0.7371 \\
\hline 2-(Tert-butylamino)ethanol & $0 \cdot 5856$ & -0.7671 & 6 & $0 \cdot 41$ & $-1 \cdot 673$ & $-0 \cdot 9764$ & $-1 \cdot 3016$ \\
\hline Diethanolamine & $0 \cdot 5880$ & -0.7685 & 4 & $-1 \cdot 43$ & $-1 \cdot 7941$ & $-1 \cdot 4275$ & $-1 \cdot 5728$ \\
\hline 1,3-Diamino-2-hydroxy-propane & $0 \cdot 6407$ & $-0 \cdot 8517$ & 3 & $-2 \cdot 05$ & -1.4275 & $-1 \cdot 6530$ & $-1 \cdot 2456$ \\
\hline N-Methyldiethanol amine & $0 \cdot 5309$ & -0.7675 & 5 & $-1 \cdot 04$ & $-1 \cdot 8338$ & $-1 \cdot 2020$ & -1.9396 \\
\hline 3-(Methylamino)-1,2-propanediol & $0 \cdot 5936$ & -0.7897 & 4 & $-1 \cdot 82$ & $-1 \cdot 5341$ & $-1 \cdot 4275$ & $-1 \cdot 5225$ \\
\hline Triethanolamine & $0 \cdot 5602$ & $-0 \cdot 7678$ & 6 & $-1 \cdot 00$ & $-1 \cdot 7488$ & $-0 \cdot 9764$ & $-1 \cdot 5298$ \\
\hline \multicolumn{8}{|l|}{ Acetylenic alcohols } \\
\hline 3-Butyn-2-ol & 0.7438 & $-0 \cdot 7525$ & 4 & $0 \cdot 14$ & $-0 \cdot 4024$ & $-0 \cdot 8795$ & -0.781 \\
\hline 1-Pentyn-3-ol & 0.7443 & -0.7565 & 5 & 0.67 & $-1 \cdot 1776$ & -0.5463 & -0.4085 \\
\hline 2-Pentyn-1-ol & 0.6737 & -0.7387 & 5 & $0 \cdot 89$ & -0.5724 & -0.5463 & -0.6729 \\
\hline 2-Penten-4-yn-1-ol & 0.6042 & -0.7593 & 6 & $-0 \cdot 01$ & -0.5549 & $-0 \cdot 2130$ & -0.5625 \\
\hline 1-Hexyn-3-ol & 0.7265 & -0.7565 & 6 & $1 \cdot 2$ & 0.6574 & $-0 \cdot 2130$ & $-0 \cdot 1044$ \\
\hline 1-Heptyn-3-ol & 0.7227 & -0.7566 & 7 & 1.73 & $-0 \cdot 265$ & $0 \cdot 1202$ & $0 \cdot 252$ \\
\hline 4-Heptyn-3-ol & $0 \cdot 6704$ & -0.7601 & 7 & 1.73 & -0.0336 & $0 \cdot 1202$ & $0 \cdot 0561$ \\
\hline 2-Octyn-1-ol & 0.6495 & -0.7388 & 8 & $2 \cdot 48$ & $0 \cdot 1944$ & 0.4534 & $0 \cdot 3485$ \\
\hline 2-Nonyn-1-ol & $0 \cdot 6487$ & -0.7388 & 9 & $3 \cdot 01$ & $0 \cdot 6486$ & 0.7867 & 0.7162 \\
\hline 2-Decyn-1-ol & $0 \cdot 6481$ & -0.7388 & 10 & $3 \cdot 54$ & 0.9855 & $1 \cdot 1199$ & 1.0847 \\
\hline 2-Tridecyn-1-ol & $0 \cdot 6474$ & -0.7388 & 13 & $5 \cdot 13$ & $2 \cdot 3665$ & $2 \cdot 1196$ & $2 \cdot 1941$ \\
\hline 4-Methyl-1-pentyn-3-ol & 0.7265 & -0.7565 & 6 & 1.07 & -0.0267 & $-0 \cdot 2130$ & $-0 \cdot 1044$ \\
\hline 4-Methyl-1-heptyn-3-ol & $0 \cdot 7018$ & $-0 \cdot 7566$ & 8 & $2 \cdot 13$ & 0.7426 & 0.4534 & $0 \cdot 5444$ \\
\hline \multicolumn{8}{|l|}{ Unsaturated alcohol } \\
\hline 2-Methyl-3-buten-2-ol & 0.6225 & -0.7821 & 5 & $0 \cdot 52$ & $-1 \cdot 3889$ & $-1 \cdot 3007$ & $-1 \cdot 2972$ \\
\hline 4-Pentyn-1-ol & 0.7562 & -0.7561 & 5 & $-0 \cdot 01$ & -1.4204 & $-1 \cdot 3007$ & $-1 \cdot 5947$ \\
\hline 2-Methyl-3-butyn-2-ol & 0.7465 & -0.7635 & 5 & $0 \cdot 28$ & $-1 \cdot 3114$ & $-1 \cdot 3007$ & $-1 \cdot 5731$ \\
\hline trans-3-Hexen-1-ol & 0.4768 & -0.7625 & 6 & 1.40 & -0.7772 & -0.8914 & $-0 \cdot 6088$ \\
\hline cis-3-Hexen-1-ol & $0 \cdot 5049$ & -0.7703 & 6 & 1.40 & $-0 \cdot 8091$ & -0.8914 & -0.6714 \\
\hline 5-Hexyn-1-ol & 0.7024 & -0.7667 & 6 & $0 \cdot 52$ & $-1 \cdot 2948$ & -0.8914 & $-1 \cdot 1108$ \\
\hline 3-Methyl-1-pentyn-3-ol & 0.7596 & -0.768 & 6 & 1.07 & $-1 \cdot 3226$ & -0.8914 & $-1 \cdot 2381$ \\
\hline 4-Hexen-1-ol & $0 \cdot 4780$ & -0.7634 & 6 & $1 \cdot 40$ & $-0 \cdot 754$ & $-0 \cdot 8914$ & $-0 \cdot 6115$ \\
\hline 5-Hexen-1-ol & $0 \cdot 5493$ & -0.7636 & 6 & 1.40 & $-0 \cdot 8411$ & $-0 \cdot 8914$ & -0.7702 \\
\hline 4-Pentyn-2-ol & 0.7275 & -0.772 & 5 & $0 \cdot 12$ & $-1 \cdot 6324$ & $-1 \cdot 3007$ & $-1 \cdot 5308$ \\
\hline 5-Hexyn-3-ol & 0.7249 & -0.7808 & 6 & 0.65 & $-1 \cdot 4043$ & $-0 \cdot 8914$ & $-1 \cdot 1609$ \\
\hline 3-Heptyn-1-ol & $0 \cdot 6046$ & -0.7656 & 7 & $1 \cdot 40$ & $-0 \cdot 3231$ & $-0 \cdot 4820$ & $-0 \cdot 5291$ \\
\hline 4-Heptyn-2-ol & $0 \cdot 6054$ & -0.7723 & 7 & $1 \cdot 18$ & $-0 \cdot 616$ & $-0 \cdot 4820$ & -0.5309 \\
\hline 3-Octyn-1-ol & $0 \cdot 5983$ & -0.7656 & 8 & 1.93 & $0 \cdot 017$ & -0.0727 & $-0 \cdot 1509$ \\
\hline 3-Nonyn-1-ol & $0 \cdot 5942$ & -0.7656 & 9 & $2 \cdot 46$ & $0 \cdot 3401$ & $0 \cdot 3366$ & $0 \cdot 2223$ \\
\hline 2-Propen-1-ol & 0.6632 & -0.7531 & 3 & $0 \cdot 17$ & -1.9178 & $-2 \cdot 1193$ & $-2 \cdot 116$ \\
\hline
\end{tabular}


Table 2. (Contd...)

\begin{tabular}{|c|c|c|c|c|c|c|c|}
\hline \multirow[b]{2}{*}{ Molecules } & \multirow[b]{2}{*}{$\omega$} & \multirow[b]{2}{*}{$Q_{k}^{\max }$} & \multirow[b]{2}{*}{$N c$} & \multirow[b]{2}{*}{$\log P^{*}$} & \multicolumn{3}{|c|}{$\mathrm{pIGC}_{50}$} \\
\hline & & & & & Experiment* & Calc. $(N c)$ & Calc. $(N c, \omega)$ \\
\hline 2-Buten-1-ol & $0 \cdot 5471$ & -0.7588 & 4 & $0 \cdot 34$ & -1.4719 & $-1 \cdot 7100$ & -1.4935 \\
\hline$(+/-)-3$-Buten-2-ol & 0.6295 & -0.771 & 4 & $0 \cdot 12$ & $-1 \cdot 0529$ & $-1 \cdot 7100$ & -1.6769 \\
\hline cis-2-Buten-1,4-diol & $0 \cdot 6479$ & -0.7578 & 5 & $-0 \cdot 81$ & $-2 \cdot 1495$ & $-1 \cdot 3007$ & $-1 \cdot 3537$ \\
\hline cis-2-Penten-1-ol & $0 \cdot 5885$ & -0.755 & 5 & $0 \cdot 87$ & $-1 \cdot 1052$ & $-1 \cdot 3007$ & $-1 \cdot 2215$ \\
\hline 3-Penten-2-ol & 0.5738 & -0.7709 & 5 & $0 \cdot 65$ & $-1 \cdot 401$ & $-1 \cdot 3007$ & $-1 \cdot 1888$ \\
\hline trans-2-hexen-1-ol & 0.4642 & -0.7591 & 6 & 1.40 & $-0 \cdot 4718$ & $-0 \cdot 8914$ & -0.5808 \\
\hline 1-Hexen-3-ol & 0.6304 & -0.7748 & 6 & $1 \cdot 18$ & $-0 \cdot 8113$ & $-0 \cdot 8914$ & -0.9506 \\
\hline cis-2-Hexen-1-ol & $0 \cdot 5381$ & -0.7588 & 6 & 1.40 & -0.7767 & $-0 \cdot 8914$ & -0.7452 \\
\hline trans-2-Octen-1-ol & 0.4621 & $-0 \cdot 759$ & 8 & $2 \cdot 45$ & $0 \cdot 3654$ & -0.0727 & $0 \cdot 1521$ \\
\hline \multicolumn{8}{|l|}{ Amines } \\
\hline Propylamine & 0.6353 & -0.8330 & 3 & 0.47 & $-0 \cdot 7075$ & $-1 \cdot 0050$ & $-1 \cdot 0027$ \\
\hline Butylamine & 0.6334 & -0.8325 & 4 & 0.97 & -0.5735 & $-0 \cdot 8910$ & -0.8908 \\
\hline$N$-Methylpropylamine & $0 \cdot 5455$ & $-0 \cdot 6865$ & 4 & $0 \cdot 84$ & $-0 \cdot 8087$ & $-0 \cdot 8910$ & -0.8759 \\
\hline Amylamine & 0.6218 & -0.8510 & 5 & 1.49 & $-0 \cdot 4848$ & -0.7780 & -0.7772 \\
\hline$N$-Methylbutylamine & $0 \cdot 5416$ & -0.6826 & 5 & $1 \cdot 33$ & -0.6784 & -0.7780 & -0.7636 \\
\hline Hexylamine & 0.6213 & $-0 \cdot 8510$ & 6 & $2 \cdot 06$ & $-0 \cdot 2197$ & $-0 \cdot 6640$ & -0.6656 \\
\hline Isopropylamine & $0 \cdot 6842$ & -0.8479 & 3 & $0 \cdot 26$ & -0.8635 & $-1 \cdot 0050$ & $-1 \cdot 0110$ \\
\hline Isobutylamine & 0.6703 & -0.8631 & 4 & 0.73 & $-0 \cdot 2616$ & -0.8910 & -0.8971 \\
\hline$N, N$-Dimethylethylamine & $0 \cdot 4764$ & -0.5751 & 4 & $0 \cdot 70$ & -0.9083 & $-0 \cdot 8910$ & $-0 \cdot 8641$ \\
\hline$(+/-)$-sec-Butylamine & 0.6626 & -0.8473 & 4 & $0 \cdot 74$ & -0.6708 & -0.8910 & -0.8958 \\
\hline Isoamylamine & $0 \cdot 6505$ & -0.8314 & 5 & $1 \cdot 32$ & -0.5774 & -0.7780 & -0.7821 \\
\hline 1-Methylbutylamine & $0 \cdot 6543$ & -0.8469 & 5 & $1 \cdot 23$ & -0.6846 & -0.7780 & -0.7828 \\
\hline 1-Ethylpropylamine & 0.6303 & -0.8455 & 7 & $1 \cdot 23$ & $-0 \cdot 8129$ & -0.5510 & -0.5555 \\
\hline 2-Methylbutylamine & 0.6449 & -0.8380 & 5 & $1 \cdot 32$ & -0.4774 & -0.7780 & -0.7812 \\
\hline$N, N$-Diethylmethylamine & $0 \cdot 4888$ & -0.5714 & 5 & 0.95 & -0.7559 & -0.7780 & -0.7546 \\
\hline tert-Butylamine & 0.7163 & -0.8541 & 4 & $0 \cdot 40$ & -0.8973 & -0.8910 & -0.9049 \\
\hline tert-Amylamine & $0 \cdot 6995$ & -0.8592 & 5 & $1 \cdot 10$ & -0.6978 & -0.7780 & -0.7904 \\
\hline$(+/-)-1,2$-Dimethylpropylamine & 0.6367 & $-0 \cdot 8457$ & 5 & $1 \cdot 10$ & -0.7095 & $-0 \cdot 7780$ & -0.7798 \\
\hline Propargylamine & $0 \cdot 6898$ & $-0 \cdot 8084$ & 3 & -0.43 & $-0 \cdot 826$ & $-1 \cdot 0050$ & $-1 \cdot 0120$ \\
\hline$N$-Methylpropargylamine & 0.6355 & -0.6632 & 4 & 0.08 & $-0 \cdot 9818$ & -0.8910 & -0.8912 \\
\hline 1-Dimethylamino-2-propyne & $0 \cdot 5750$ & -0.5392 & 5 & $-0 \cdot 01$ & $-1 \cdot 1451$ & -0.7780 & -0.7693 \\
\hline 1,1-Dimethylpropargylamine & $0 \cdot 6681$ & -0.8289 & 5 & $0 \cdot 64$ & $-0 \cdot 9104$ & -0.7780 & -0.7851 \\
\hline 2-Methoxyethylamine & $0 \cdot 6585$ & $-0 \cdot 8568$ & 3 & -0.67 & $-1 \cdot 7903$ & $-1 \cdot 0050$ & $-1 \cdot 0067$ \\
\hline 3-Methoxypropylamine & $0 \cdot 6608$ & -0.8478 & 4 & $-1 \cdot 02$ & -1.7725 & $-0 \cdot 8910$ & -0.8955 \\
\hline 3-Ethoxypropylamine & 0.6592 & -0.8479 & 5 & -0.49 & -1.7027 & -0.7780 & -0.7836 \\
\hline
\end{tabular}

*Taken from reference 10

dicted toxicity trend is satisfactory when compared with the observed one, for the individual outlier molecules it is difficult to provide with a rationale $a$ priori. It may be noted that the calculated $\mathrm{pIGC}_{50}$ values plotted in figure 1 are obtained through different regression models for 13 different sets of molecules. In each set the molecules of similar chemical behaviour are included. In case we take all the molecules together the following regression equations are obtained:

$$
\begin{aligned}
\mathrm{pIGC}_{50} & =0.2789 \times N_{\mathrm{C}}-2 \cdot 2484 \\
R & =0 \cdot 805 ; \mathrm{SD}=0.551 ; N=252 .
\end{aligned}
$$

$$
\begin{aligned}
\mathrm{pIGC}_{50} & =0.2838 \times N_{C}+0.6415 \times \omega-2.7888 \\
R & =0.812 ; \mathrm{SD}=0.542 ; N=252 .
\end{aligned}
$$

It may be noted that for a diverse class of chemical compounds $N_{\mathrm{C}}$ and $\omega$ may still be considered to be useful descriptors. Corresponding plots are provided in figure $2 \mathrm{a}, \mathrm{b}$. The correlation improves further in case a couple of sets are removed as was done by Schultz et $a l^{13}$. For the sake of completeness we also include the plot of the experimental toxicity with $\log P$ for the same 252 molecules (figure 2c). The correlation is comparable to that obtained in figure 2a which is expected because of the inter cor- 
Table 3. Regression models for different groups of aliphatic compounds for estimating their toxicity towards Tetrahymena pyriformis

\begin{tabular}{|c|c|c|c|}
\hline Molecules & Regression equations & $\mathrm{R}$ & SD \\
\hline \multicolumn{4}{|l|}{ Aliphatic electron acceptors } \\
\hline \multirow[t]{4}{*}{ Diols $(N=10)$} & $\mathrm{pIGC}_{50}=0.4497 \times N_{\mathrm{C}}-4.0855$ & $0 \cdot 9683$ & $0 \cdot 2781$ \\
\hline & $\mathrm{pIGC}_{50}=0.8059 \times \log P-1.4688$ & $0 \cdot 9892$ & $0 \cdot 1617$ \\
\hline & $\mathrm{pIGC}_{50}=-12.4224 \times \omega+0.3554 \times N_{\mathrm{C}}+7.6094$ & $0 \cdot 9826$ & $0 \cdot 2070$ \\
\hline & $\mathrm{pIGC}_{50}=10.0678 \times \omega+0.9625 \times \log P-10.5043$ & 0.9934 & $0 \cdot 1270$ \\
\hline \multirow{4}{*}{$\begin{array}{l}\text { Halogenated alcohols } \\
\qquad(N=11)\end{array}$} & $\mathrm{pIGC}_{50}=0.3271 \times N_{\mathrm{C}}-2.0248$ & $0 \cdot 8923$ & $0 \cdot 3852$ \\
\hline & $\mathrm{pIGC}_{50}=0.7783 \times \log P-1.3735$ & $0 \cdot 9486$ & $0 \cdot 2561$ \\
\hline & $\mathrm{pIGC}_{50}=-6.2863 \times \omega+0.1982 \times N_{C}+4.5793$ & 0.9424 & $0 \cdot 2855$ \\
\hline & $\mathrm{pIGC}_{50}=-4.0784 \times \omega+0.5772 \times \log P+2.7468$ & 0.9646 & $0 \cdot 2169$ \\
\hline \multirow[t]{4}{*}{ Saturated alcohols $(N=32)$} & $\mathrm{pIGC}_{50}=0.4144 \times N_{\mathrm{C}}-3.0801$ & 0.9634 & $0 \cdot 3456$ \\
\hline & $\mathrm{pIGC}_{50}=0.7745 \times \log P-2.0034$ & $0 \cdot 9903$ & $0 \cdot 1777$ \\
\hline & $\mathrm{pIGC}_{50}=0.8927 \times \omega+0.4261 \times N_{C}-3.9484$ & 0.9636 & $0 \cdot 3451$ \\
\hline & $\mathrm{pIGC}_{50}=1.6835 \times \omega+0.8138 \times \log P-3.5796$ & 0.9907 & $0 \cdot 1739$ \\
\hline \multirow[t]{4}{*}{ Carboxylic acids $(N=28)$} & $\mathrm{pIGC}_{50}=0.1116 \times N_{\mathrm{C}}-0.9678$ & $0 \cdot 6676$ & $0 \cdot 2917$ \\
\hline & $\mathrm{pIGC}_{50}=0.2857 \times \log P-0.7006$ & 0.9586 & $0 \cdot 1070$ \\
\hline & $\mathrm{pIGC}_{50}=-5.4426 \times \omega+0.0338 \times N_{\mathrm{C}}+4.8562$ & $0 \cdot 8801$ & $0 \cdot 1860$ \\
\hline & $\mathrm{pIGC}_{50}=-0.3944 \times \omega+0.2715 \times \log P-0.2924$ & 0.9589 & $0 \cdot 1066$ \\
\hline \multirow[t]{4}{*}{ Halogenated acids $(N=11)$} & $\mathrm{pIGC}_{50}=0.2257 \times N_{\mathrm{C}}-1.3481$ & 0.6564 & $0 \cdot 3632$ \\
\hline & $\mathrm{pIGC}_{50}=0.4620 \times \log P-0.8744$ & $0 \cdot 8107$ & $0 \cdot 2285$ \\
\hline & $\mathrm{pIGC}_{50}=1.8930 \times \omega+0.0976 \times N_{\mathrm{C}}-2.2827$ & 0.9186 & $0 \cdot 1903$ \\
\hline & $\mathrm{pIGC}_{50}=1.6012 \times \omega+0.2001 \times \log P-1.8388$ & 0.9169 & $0 \cdot 1762$ \\
\hline \multirow[t]{4}{*}{ Mono esters $(N=31)$} & $\mathrm{pIGC}_{50}=0.3645 \times N_{\mathrm{C}}-2.7969$ & 0.9189 & $0 \cdot 3710$ \\
\hline & $\mathrm{pIGC}_{50}=0.7599 \times \log P-2.0274$ & 0.9645 & $0 \cdot 2396$ \\
\hline & $\mathrm{pIGC}_{50}=-10.9131 \times \omega+0.2554 \times N_{\mathrm{C}}+8.1384$ & 0.9352 & $0 \cdot 3330$ \\
\hline & $\mathrm{pIGC}_{50}=-3.0902 \times \omega+0.6960 \times \log P+1.0027$ & $0 \cdot 9655$ & $0 \cdot 2365$ \\
\hline \multirow[t]{4}{*}{ Diesters $(N=20)$} & $\mathrm{pIGC}_{50}=0.2861 \times N_{\mathrm{C}}-2.884$ & 0.9299 & $0 \cdot 3382$ \\
\hline & $\mathrm{pIGC}_{50}=0.6338 \times \log P-1.3322$ & 0.9539 & $0 \cdot 2632$ \\
\hline & $\mathrm{pIGC}_{50}=-4.8166 \times \omega+0.1999 \times N_{\mathrm{C}}+1.1227$ & 0.9636 & $0 \cdot 2460$ \\
\hline & $\mathrm{pIGC}_{50}=-4.2407 \times \omega+0.4687 \times \log P+1.7763$ & 0.9790 & $0 \cdot 1834$ \\
\hline \multirow[t]{4}{*}{ Aldehydes $(N=13)$} & $\mathrm{pIGC}_{50}=0.2230 \times N_{C}-1.4027$ & $0 \cdot 8980$ & $0 \cdot 2459$ \\
\hline & $\mathrm{pIGC}_{50}=0.4628 \times \log P-0.8864$ & 0.9227 & $0 \cdot 1988$ \\
\hline & $\mathrm{pIGC}_{50}=-2.5248 \times \omega+0.1228 \times N_{C}+1.3002$ & 0.9332 & $0 \cdot 2008$ \\
\hline & $\mathrm{pIGC}_{50}=-2.1731 \times \omega+0.2904 \times \log P+1.2280$ & $0 \cdot 9496$ & $0 \cdot 1664$ \\
\hline \multirow[t]{4}{*}{ Ketones $(N=15)$} & $\mathrm{pIGC}_{50}=0.4147 \times N_{\mathrm{C}}-3.4470$ & 0.9850 & $0 \cdot 2249$ \\
\hline & $\mathrm{pIGC}_{50}=0.7720 \times \log P-2.0314$ & 0.9872 & $0 \cdot 2048$ \\
\hline & $\mathrm{pIGC}_{50}=-3.2176 \times \omega+0.38989 \times N_{\mathrm{C}}-0.6459$ & 0.9855 & $0 \cdot 2211$ \\
\hline & $\mathrm{pIGC}_{50}=-1.4487 \times \omega+0.7511 \times \log P-0.8080$ & 0.9873 & $0 \cdot 2041$ \\
\hline \multicolumn{4}{|l|}{ Aliphatic electron donors } \\
\hline \multirow[t]{4}{*}{ Amino alcohols $(N=18)$} & $\mathrm{pIGC}_{50}=0.2255 \times N_{\mathrm{C}}-2.3296$ & $0 \cdot 4711$ & $0 \cdot 4596$ \\
\hline & $\mathrm{pIGC}_{50}=0.3533 \times \log P-1.0529$ & $0 \cdot 5829$ & $0 \cdot 2468$ \\
\hline & $\mathrm{pIGC}_{50}=8.9875 \times \omega+0.1464 \times N_{\mathrm{C}}-7.4431$ & 0.9152 & $0 \cdot 2100$ \\
\hline & $\mathrm{pIGC}_{50}=8.5520 \times \omega+0.2282 \times \log P-6.3481$ & 0.9377 & $0 \cdot 1697$ \\
\hline \multirow[t]{4}{*}{ Acetylenic alcohols $(N=13)$} & $\mathrm{pIGC}_{50}=0.3332 \times N_{\mathrm{C}}-2.2125$ & $0 \cdot 8942$ & $0 \cdot 4218$ \\
\hline & $\mathrm{pIGC}_{50}=0.5506 \times \log P-0.8071$ & $0 \cdot 8842$ & $0 \cdot 3891$ \\
\hline & $\mathrm{pIGC}_{50}=3.7452 \times \omega+0.3707 \times N_{\mathrm{C}}-5.0494$ & $0 \cdot 9080$ & $0 \cdot 3947$ \\
\hline & $\mathrm{pIGC}_{50}=0.2523 \times \omega+0.5538 \times \log P-0.9858$ & $0 \cdot 8843$ & $0 \cdot 3890$ \\
\hline \multirow{4}{*}{$\begin{array}{l}\text { Unsaturated alcohols } \\
\qquad(N=25)\end{array}$} & $\mathrm{pIGC}_{50}=0.4093 \times N_{\mathrm{C}}-3.3473$ & $0 \cdot 8580$ & $0 \cdot 3311$ \\
\hline & $\mathrm{pIGC}_{50}=0.7587 \times \log P-1.6861$ & 0.9315 & $0 \cdot 2185$ \\
\hline & $\mathrm{pIGC}_{50}=-2.2250 \times \omega+0.3641 \times N_{\mathrm{C}}-1.7327$ & 0.9136 & $0 \cdot 2622$ \\
\hline & $\mathrm{pIGC}_{50}=-0.0271 \times \omega+0.7568 \times \log P-1.6679$ & 0.9315 & $0 \cdot 2185$ \\
\hline \multirow[t]{6}{*}{ Amines $(N=25)$} & $\mathrm{pIGC}_{50}=0.1136 \times N_{\mathrm{C}}-1.3456$ & $0 \cdot 2711$ & $0 \cdot 3965$ \\
\hline & $\mathrm{pIGC}_{50}=0.4609 \times \log P-1.1380$ & 0.8534 & $0 \cdot 1833$ \\
\hline & $\mathrm{pIGC}_{50}=-0.1700 \times \omega+0.1116 \times N_{\mathrm{C}}-1.2295$ & $0 \cdot 2723$ & $0 \cdot 3964$ \\
\hline & $\mathrm{pIGC}_{50}=0.9307 \times \omega+0.4792 \times \log P-1.7367$ & $0 \cdot 8641$ & $0 \cdot 1792$ \\
\hline & $\mathrm{pIGC}_{50}=0.1162 \times Q_{k}^{\max }+2.1524 \times \omega_{\max }^{-}+0.0669 \times N_{\mathrm{C}}-1.8782$ & $0 \cdot 8692$ & $0 \cdot 2037$ \\
\hline & $\mathrm{pIGC}_{50}=0.0681 \times Q_{k}^{\max }+1.3490 \times \omega_{\max }^{-}+0.2802 \times \log P-1.4885$ & 0.9429 & $0 \cdot 1293$ \\
\hline
\end{tabular}



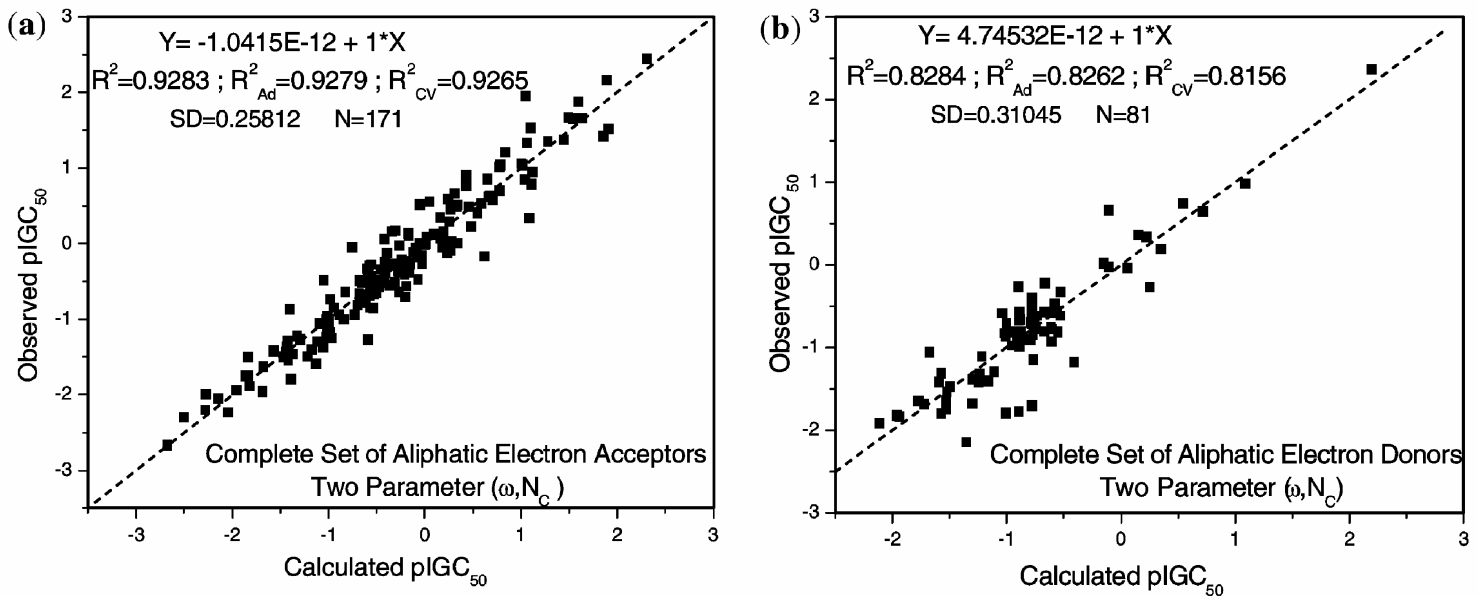

Figure 1. Observed versus calculated $\mathrm{pIGC}_{50}$ values using two-parameter $\left(\omega, N_{\mathrm{C}}\right)$ regression models for the (a) Complete set of aliphatic electron acceptors and (b) Complete set of aliphatic electron donors.

relation between $\log P$ and $N_{\mathrm{C}}$ (figure $2 \mathrm{~d}$ ). It may be noted that unlike $\log P, N_{\mathrm{C}}$ does not require any software (instrument) to compute it (determine it experimentally).

It may be noted that the macroscopic descriptors like $\log P$ or $N_{\mathrm{C}}$ would be useful for a broad spectrum of systems. However, electronic descriptors like $\omega$ would be useful when systems with similar electronic environment are analysed. They would be specially useful when molecules will have nearly identical $\log P\left(N_{\mathrm{C}}\right)$ values. For molecules with similar electronic environment local (or group) electrophilicity would highlight the importance of the site (group) especially responsible for the toxic behaviour.

It is important to mention that one should be careful in analysing a figure containing different models. When the models for separate groups of congener molecules are also analysed it becomes transparent. When a single model is used the correlation becomes at best the mediocre which is expected because two molecules belonging to two completely different classes (say an amine and a carboxylic acid) may not behave in a similar fashion. Another aspect one must be careful about is the false correlation resulting out of randomization which happens in certain cases in the present analysis as well. On an average the $N_{C}$ based models may be considered to be good starting points (without any experiment/ computation) for building up more reasonable ones.

\section{Concluding remarks}

The number of atoms in a molecule can provide important insights into its possible toxic behaviour. It can be used as a molecular descriptor for predicting $\mathrm{pIGC}_{50}$ values of various aliphatic compounds against the ciliate $T$. pyriformis. Considering the simplicity of this descriptor as opposed to $\log \mathrm{P}$ it is quite gratifying to note that the former can be considered to be a crude approximation to the latter. The situation improves further when electrophilicity is used as an additional descriptor. Although the calculation of electrophilicity index $(\omega)$ requires high level computation its use becomes mandatory in certain cases, e.g. halogenated alcohols, carboxylic acids, halogenated acids, aldehydes, amino alcohols and unsaturated alcohols. Local electro (nucleo) philicity and atomic charges are also considered to take care of local soft-soft and hard-hard interactions, which resulted in robust three-parameter QSTR models. Conceptual DFT based descriptors have helped in many ways to understand the structure of molecules and their reactivity. In this regard it is necessary to mention that development of conceptual DFT has revolutionized the various aspects of chemical reactivity by providing strong foundations for the qualitative concepts. With the help of global and local reactivity descriptors, it is now possible to analyse the chemical reactivity of the whole molecule as well as the site selectivity of an atom in it. Experimental activity can be obtained in many different ways/sources and it is difficult to generate a general correlation which is the major limiting step for any QSAR/QSTR/QSPR study. The usefulness of these chemical reactivity descriptors in the quantitative structure activity/reactivity/toxicity parlance has been demonstrated. The developed model has greater flexibility in the sense that it can be extended further. This may save time and money that is, being spent 

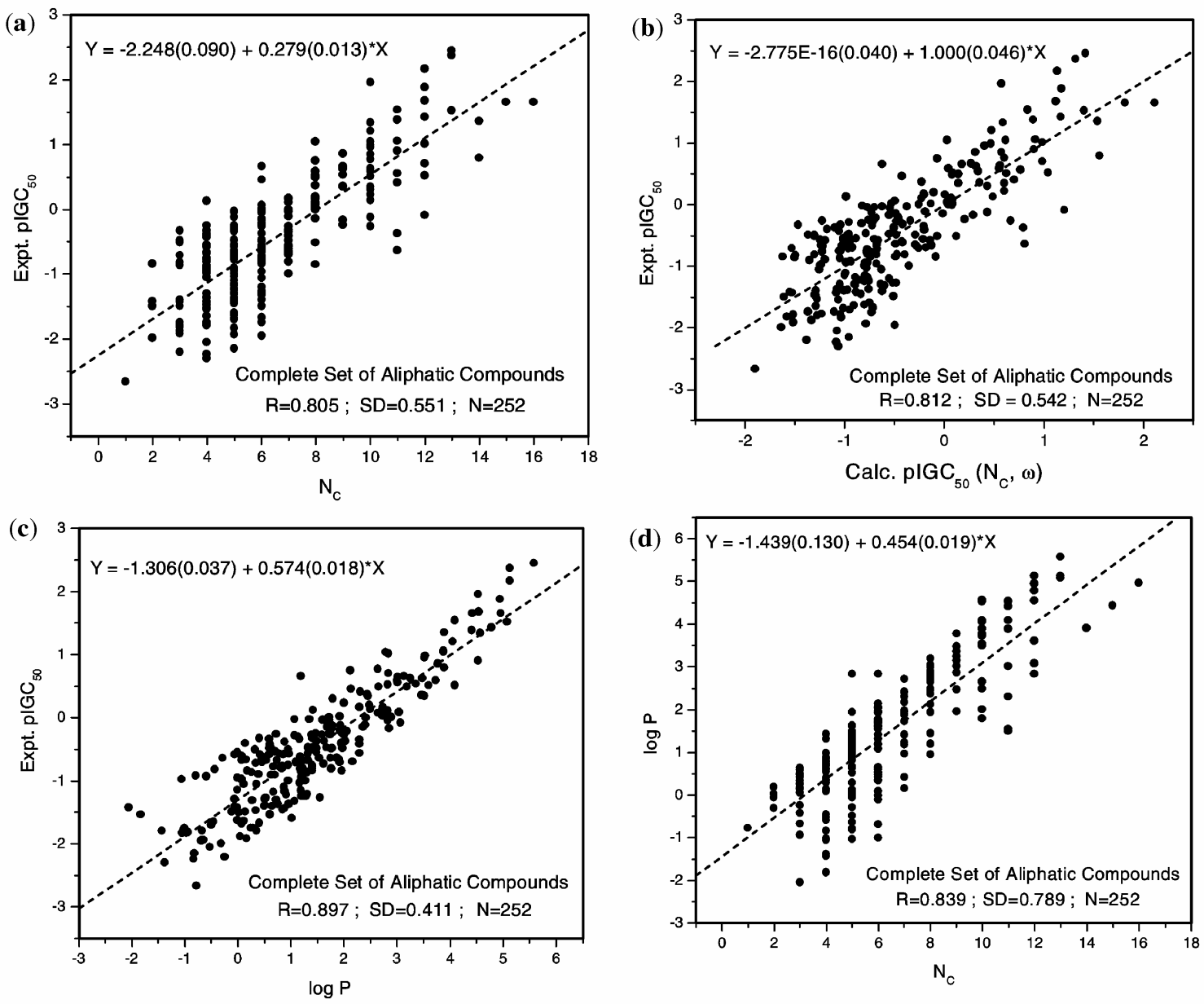

Figure 2. Observed pIGC ${ }_{50}$ versus the (a) Number of carbon atoms $\left(N_{\mathrm{C}}\right)$, (b) Calculated pIGC (b0 $_{50}$ values using two-parameter $\left(\omega, N_{\mathrm{C}}\right)$ regression model and (c) $\log P$ along with the (d) Inter-correlation between $\log P$ and $N_{\mathrm{C}}$ for the complete set of 252 aliphatic compounds.

on carrying out experiments. The developed theoretical models along with experiment can always be utilized to arrive at the best possible solution for any future drug discovery.

\section{Acknowledgements}

This paper is dedicated to Professor Debashis Mukherjee on his 60th birthday. We thank BRNS, Mumbai for financial assistance and the referee for constructive criticism. P B acknowledges the fund for Scientific Research-Flanders (FWO-Vlaanderen) for continuous support for his group.

\section{References}

1. Smeyers Y G, Bouniam L, Smeyers N J, Ezzamarty A, Hernandez-Laguna A and Sainz-Diaz C I 1998 Eur. J. Med. Chem. 33103
2. Busse W D, Ganellin C R and Mitscher L A 1996 Eur. J. Med. Chem. 31747

3. Hansch C, Hoekman D, Leo A, Weininger D and Selassie C 2002 Chem. Rev. 102783

4. Hansch C, Kurup A, Garg R and Gao H 2001 Chem. Rev. 101619

5. Hansch C, Maloney P P, Fujita T and Muir R M 1962 Nature 194178

6. Hansch C, Li R I, Blaney J M and Langridge R J 1982 J. Med. Chem. 25777

7. Leach A R and Gillet V J 2003 An introduction to chemoinformatics (Kluwer: Dordrecht)

8. Ormerod A, Willett P and Bawden D 1989 Quant. Struct-Act. Relat. 8115

9. Akers K S, Sinks G D and Schultz T W 1999 Environ. Toxicol. Pharmacol. 733

10. Schultz T W 1997 Toxicol. Methods 7289. TETRATOX database; http://www.vet.utk.edu/ TETRATOX/

11. Dimitrov S D, Mekenyan O G, Sinks G D and Schultz T W 2003 J. Mol. Struct. (THEOCHEM) 622 63 
12. Netzeva T I, Schultz T W, Aptula A O and Cronin M T D 2003 SAR QSAR Environ. Res. 14265

13. Schultz T W, Cronin M T D, Netzeva T I and Aptula A O 2002 Chem. Res. Tox. 151602

14. Cronin M T D, Manga N, Seward J R, Sinks G D and Schultz T W 2001 Chem. Res. Tox. 141498

15. Xue Y, Li H, Ung C Y, Yap C W and Chen Y Z 2006 Chem. Res. Tox. 191030

16. (a) Toropov A A and Benfenati E 2004 J. Mol. Struct. (THEOCHEM) 679 225; (b) Castro E A, Toropov A A, Nesterova A I and Nazarov A U $2003 \mathrm{~J}$. Mol. Struct. (THEOCHEM) 639129

17. (a) Parr R G and Yang W 1989 Density functional theory of atoms and molecules (Oxford, UK: Oxford University Press); (b) Chattaraj P K (ed.) 2005 Special issue on chemical reactivity J. Chem. Sci. 117; (c) Geerlings P, De Proft F and Langenaeker W 2003 Chem. Rev. 1031793

18. Chattaraj P K, Nath S and Maiti B 2004 Reactivity descriptors in computational medicinal chemistry for drug discovery (eds) J Tollenaere, P Bultinck, H D Winter and W Langenaeker (New York: Marcel Dekker) Chapter 11, p 295

19. Parthasarthi R, Subramanian V, Roy D R and Chattaraj P K 2004 Bioorg. Med. Chem. 125533

20. Roy D R, Parthasarathi R, Maiti B, Subramanian V and Chattaraj P K 2005 Bioorg. Med. Chem. 133405

21. Parthasarathi R, Elango M, Padmanabhan J, Subramanian V, Roy D R, Sarkar U and Chattaraj P K 2006 Indian J. Chem. A 45111

22. Parthasarathi R, Padmanabhan J, Subramanian V, Maiti B and Chattaraj P K 2004 Curr. Sci. 86535

23. Sarkar U, Roy D R, Chattaraj P K, Parthasarathi R, Padmanabhan J and Subramanian V 2005 J. Chem. Sci. 117599

24. Padmanabhan J, Parthasarathi R, Subramanian V and Chattaraj P K 2006 Chem. Res. Tox. 19356

25. Roy D R, Parthasarathi R, Padmanabhan J, Sarkar U, Subramanian V and Chattaraj P K 2006 J. Phys. Chem. A 1101084.

26. Parthasarathi R, Padmanabhan J, Subramanian V, Maiti B and Chattaraj P K 2003 J. Phys. Chem. A 10710346

27. Padmanabhan J, Parthasarathi R, Subramanian V and Chattaraj P K 2005 J. Phys. Chem. A 10911043
28. Padmanabhan J, Parthasarathi R, Subramanian V and Chattaraj P K 2006 J. Phys. Chem. A 1102739

29. Roy D R, Sarkar U, Chattaraj P K, Mitra A, Padmanabhan J, Parthasarathi R, Subramanian V, Vandamme S and Bultinck P 2006 Mol. Div. 10119

30. Roy D R, Parthasarathi R, Subramanian V and Chattaraj P K 2006 QSAR \& Comb. Sci. 25114

31. Parr R G, Szentpaly L V and Liu S 1999 J. Am. Chem. Soc. 1211922

32. Chattaraj P K and Roy D R 2007 Chem. Rev. 107 2065

33. Chattaraj P K, Maiti B and Sarkar U 2003 J. Phys. Chem. A 1074973

34. (a) Szymanski V, Müller W R, Knop J V and Trinajstić N 1984 Int. J. Quantum Chem. 30 173; (b) Roy D R, Pal N, Mitra A, Bultinck P, Parthasarathi R, Subramanian V and Chattaraj P K 2007 Eur. J. Med. Chem. Doi:10:10.1016/j.ejmech.2007.01.028; (c) Giri S, Roy D R, Bultinck P, Subramanian V and Chattaraj P K 2007 QSAR Comb. Sci. (in press)

35. Yang W and Mortier W J $1986 \mathrm{~J}$. Am. Chem. Soc. 1085708

36. Klopman G (ed.) 1974 Chemical reactivity and reaction paths (New York: Wiley); Klopman G $1968 \mathrm{~J}$. Am. Chem. Soc. 90223

37. Chattaraj P K 2001 J. Phys. Chem. A 105511

38. Melin J, Aparicio F, Subramanian V, Galvan M and Chattaraj P K 2004 J. Phys. Chem. A 1082487

39. Gaussian 03, Revision B.03; Pittsburgh, PA: Gaussian, Inc.

40. Livingstone D J and Salt D W 2006 Rev. Comput. Chem. 21287

41. Whitley D, Ford M G and Livingstone D J $2004 \mathrm{~J}$. Chem. Inf. Comp. Sci. 401160

42. Van Damme S and Bultinck P 2007 J. Comp. Chem. 281924

43. Hawkins D M, Basak S C and Mills D 2003 J. Chem. Inf. Comput. Sci. 43579

44. Principle of Parsimony: All things being approximately equal, one should accept the simplest possible model (Occam's Razor; William of Ockham, 12851349, English philosopher and logician)

45. Topliss J G and Edwards R P 1979 J. Med. Chem. 22 1238 